\title{
Digital imaging: a unified topological framework
}

\author{
Loïc Mazo - Nicolas Passat - Michel Couprie · Christian Ronse
}

August 29th, 2010

\begin{abstract}
In this article, a tractable modus operandi is proposed to model a (binary) digital image (i.e., an image defined on $\mathbb{Z}^{n}$ and equipped with a standard pair of adjacencies) as an image defined in the space of cubical complexes $\left(\mathbb{F}^{n}\right)$. In particular, it is shown that all the standard pairs of adjacencies (namely the $(4,8)$ and $(8,4)$-adjacencies in $\mathbb{Z}^{2}$, the $(6,18),(18,6),(6,26)$, and $(26,6)$-adjacencies in $\mathbb{Z}^{3}$, and more generally the $\left(2 n, 3^{n}-1\right)$ and $\left(3^{n}-1,2 n\right)$-adjacencies in $\mathbb{Z}^{n}$ ) can then be correctly modelled in $\mathbb{F}^{n}$. Moreover, it is established that the digital fundamental group of a digital image in $\mathbb{Z}^{n}$ is isomorphic to the fundamental group of its corresponding image in $\mathbb{F}^{n}$, thus proving the topological correctness of the proposed approach. From these results, it becomes possible to establish links between topology-oriented methods developed either in classical digital spaces $\left(\mathbb{Z}^{n}\right)$ or cubical complexes $\left(\mathbb{F}^{n}\right)$, and to potentially unify some of them.
\end{abstract}

Keywords digital imaging · digital topology $\cdot$ cubical complexes · homotopy $\cdot$ fundamental group

\section{Introduction}

The rapid and important rise of digital imaging, and the associated need of efficient image analysis tools for 2-D, and later 3-D (and even 4-D) digital images have provided a strong motivation to research related to the definition of

Loïc Mazo, Nicolas Passat, Christian Ronse

Université de Strasbourg, LSIIT, UMR CNRS 7005, France

Tel.: +33-368854413

Fax: +33-368854455

E-mail: loic.mazo@unistra.fr

Loïc Mazo, Michel Couprie

Université Paris-Est, Laboratoire d'Informatique Gaspard-Monge, Équipe A3SI, ESIEE Paris, France sound digital topological models. Indeed, in order to segment, process, or analyse digital images in various application fields, it is often fundamental to be able to preserve, get back or integrate topological information.

Basically, an $n$-dimensional (digital) binary image can reasonably be considered as a subset of $\mathbb{Z}^{n}$ or, equivalently, as a function from $\mathbb{Z}^{n}$ to the set of values $\{0,1\}$. However, the actual structures visualised in such images generally correspond to objects of the real world, and are thus continuous (at least at the macroscopic scale where they are considered). Consequently, they are objects of $\mathbb{R}^{n}$, and not of $\mathbb{Z}^{n}$.

In order to deal with this continuous/discrete issue, research efforts have essentially focused (since the first works, proposed nearly fifty years ago [1,2]) on specific and pragmatic questions related to topology, namely the definition of a notion of adjacency relation, and the induced notions of connectivity and arcs. These notions lead, in particular, to high-level concepts of topology, such as homotopy, with natural applications to "homotopy type-preserving" image processing.

The first solution proposed to model the topology of a digital image in $\mathbb{Z}^{n}$ was to consider that two points (also called xels) are adjacent if there are neighbours in the $n$ $\mathrm{D}$ cubic grid naturally induced by $\mathbb{Z}^{n}$ (possibly enriched by some well chosen sets of "diagonals"). In this framework, partial solutions have been found to model the above topological properties, for instance by defining dual adjacencies for the object (composed of the xels of value 1) and the background (composed of the xels of value 0) [2], thus enabling to define, from these adjacency relations, the notions of connectivity [3] and of digital fundamental group [4], which permits to compare objects from a topological point of view. This historical approach is known as digital topology [5].

Other kinds of discrete spaces, enabling to model more explicitly the continuous topological properties of digital images, have also been proposed. Among these alternative 
approaches of topology modelling, one can cite connected ordered topological spaces [6], abstract cell complexes [7] (which are globally equivalent) or orders [8]. Broadly speaking, they propose to put some "topological glue" between the xels of digital images in order to better define the topological links with their continuous analogues.

By comparison to these (more sophisticated) approaches, digital topology - proposed directly on $\mathbb{Z}^{n}$ - may appear as the less satisfactory solution to accurately deal with topological properties of binary images. Nevertheless, digital topology remains the most commonly used framework for developing image processing tools dealing with topological issues. Indeed, since digital topology is directly defined in $\mathbb{Z}^{n}$, methods relying on it will also provide final results in $\mathbb{Z}^{n}$ (namely, the actual support of the processed images), which generally constitutes a desired property in most applications. Moreover, a large literature has already been devoted to homotopy-type preservation in digital topology, especially thanks to the concept of simple point [9-12]. In this context, only very few methods have been based on alternative models (e.g., for image segmentation based on cubical complexes [13] or orders [14]; parallel skeletonisation based on cubical complexes [15]), while digital topology has led to the design of quite numerous methods, devoted (non exhaustively) to deformable models (see, e.g., $[16,17]$ ), segmentation (see, e.g., [18, 19]), image correction (see, e.g., [20,21]), or image warping (see, e.g., [22]).

Because of this intensive use of digital topology, it may be important to guarantee that there exists an actual compatibility between digital topology and the other proposed topological approaches (and more generally with the "continuous" topology). This requires, in particular, to be able to embed a binary image initially defined in $\mathbb{Z}^{n}$ into a richer space (while respecting the chosen adjacencies in $\mathbb{Z}^{n}$ ) while preserving certain topological characteristics of objects (see, e.g., $[23,24])$.

The "richer space" that is used here is $\mathbb{F}^{n}$, namely the space of cubical complexes, which is together a connected ordered topological space, a cellular space and an order (i.e., a poset). Though it is commonly admitted that there exists a strong link between digital topology and cubical complexes [25], since complexes are closed objects, the images handled in $\mathbb{F}^{n}$ correspond generally to images defined in $\mathbb{Z}^{n}$ with a $\left(3^{n}-1,2 n\right)$-adjacency pair. In [26], a method is proposed to include and improve digital topology in the framework of posets, but the case of the $(6,18)$ - and $(18,6)$-adjacency pairs is not considered. In [24,27,28], the authors give a way to embed digital pictures in a space of complexes according to the kind of connectivity that has been chosen in $\mathbb{Z}^{n}$. However, they do not use an intrinsic topology on complexes which are just a step between $\mathbb{Z}^{n}$ and $\mathbb{R}^{n}$. This leads them to define specific notions of connectedness and digital homotopy in $\mathbb{F}^{n}$. Thereby, in this article, we propose a complete framework to correctly embed a binary digital image in the topological space $\mathbb{F}^{n}$, according to the choice of adjacencies which has been made in $\mathbb{Z}^{n}$.

The sequel of the article is organised as follows. Section 2 recalls background notions and useful notations. Section 3 describes the mapping enabling to associate a binary digital image defined on $\mathbb{Z}^{n}$ to an equivalent image defined in the space of cubical complexes $\left(\mathbb{F}^{n}\right)$. A detailed study of such images is then proposed. Section 4 , which is devoted to homotopy in binary images, presents the main contribution of this work. It provides the proof that the connected components and the digital fundamental group of the digital images in $\mathbb{Z}^{n}$ are preserved in $\mathbb{F}^{n}$ when using the mapping described in Section 3. Section 5 concludes this article by summarising the contributions and presenting further works. Auxiliary properties (used in the proofs of Sections 3 and 4) are proposed in Appendix A.

\section{Background notions}

This section provides the minimal set of background notions required to make this article globally self-contained, and then more comprehensible for the reader. Some of them are standard notions of lattice theory (Section 2.1) and can also be found in $[29,30]$. The reader interested in topological notions (Section 2.2) can find complementary information in any lecture book on general topology [31,32] or on algebraic topology [33-35]. The main cubical complexes notions (Section 2.3) are described, e.g., in [15,36], while a good introduction to digital topology may be found in [5].

\subsection{Posets}

Let $X$ be a set. A binary relation on $X$ is a partial order if it is reflexive, antisymmetric, and transitive. A partially ordered set (or poset) is an ordered pair $(X, \leqslant)$ where the relation $\leqslant$ is a partial order on $X$. We write $x<y$ when $x \leqslant y$ and $x \neq y$. The relation $\geqslant$ defined on $X$ by $x \geqslant y$ if $y \leqslant x$ is a partial order on $X$ called the dual order. The covering relation $\prec$, associated to $\leqslant$, is defined by: $x<y$ (say " $y$ covers $x$ ") if $x<y$ and there is no $z$ such that $x<z<y$. We set:

$$
\begin{aligned}
x^{\uparrow} & =\{y \in X \mid x \leqslant y\} ; \\
x^{\uparrow+} & =\left\{y \in x^{\uparrow} \mid y^{\uparrow}=\{y\}\right\} ; \\
x^{\downarrow} & =\{y \in X \mid y \leqslant x\} ; \\
x^{\downarrow \star} & =x^{\downarrow} \backslash\{x\}=\{y \in X \mid y<x\} ; \\
x^{\prec} & =\{y \in X \mid x<y\} .
\end{aligned}
$$

An element $x \in X$ is minimal if $x^{\downarrow}=\{x\}$ and maximal if $x^{\uparrow}=\{x\}$. An element $x \in X$ is the minimum of $X$ if $x^{\uparrow}=X$ and is the maximum of $X$ if $x^{\downarrow}=X$. If the minimum (resp., the maximum) exists, then it is unique. 


\subsection{Topological spaces}

Let $X$ be a set, the elements of which will be called points. A topology on $X$ is a collection $\mathcal{U}$ of subsets of $X$, called open sets, such that:

(i) $\emptyset, X$ are open sets;

(ii) any finite intersection of open sets is an open set;

(iii) any union of open sets is an open set.

The complement in $X$ of an open set is called a closed set. From the above definition, any finite union of closed sets is a closed set and any intersection of closed sets is a closed set. A set of open sets is a basis for a topology if any open set is a union of open sets of this basis. A neighbourhood of a point $x \in X$ is a subset of $X$ including an open set containing $x$.

Let $(X, \leqslant)$ be a poset. The set $\mathcal{U}$ defined by $\mathcal{U}=\{U \subseteq$ $\left.X \mid \forall x \in U, x^{\uparrow} \subseteq U\right\}$ is a topology on $X$ which is called the Alexandroff topology. In this topology, the set $x^{\uparrow}$ is the smallest open set containing $x$ (or the smallest neighbourhood of $x$, called the star of $x$ ) and the set $x^{\downarrow}$ is the smallest closed set containing $x$ (the closure of $x$ ). Two points $x, y \in X$ are adjacent if $x \leqslant y$ or $y \leqslant x$. A sequence $\left(z_{i}\right)_{i=0}^{r}$ $(r \geq 0)$ of elements of $X$ is an arc in $X$ (from $z_{0}$ to $z_{r}$ ) if for all $i \in \llbracket 1, r \rrbracket, z_{i-1}$ and $z_{i}$ are distinct and adjacent ${ }^{1}$. A subset $Y$ of $X$ is connected if for all $x, y \in Y$, there exists an arc in $Y$ from $x$ to $y^{2}$. A connected component of a subset $Y$ of $X$ is a maximal (for inclusion) connected subset of $Y$.

The closure $Y^{\downarrow}$ of a subset $Y \subseteq X$ is the smallest closed set including $Y$. The interior $Y^{\circ}$ of a subset $Y \subseteq X$ is the largest open set included in $Y$ (it is also the union of all open sets included in $Y$ ). Closure and interior are dual notions since $\neg\left(Y^{\circ}\right)=(\neg Y)^{\downarrow}$ and $\neg\left(Y^{\downarrow}\right)=(\neg Y)^{\circ}$ where $\neg Y=X \backslash Y$. An open set $Y$ is a regular open set if $Y=\left(Y^{\downarrow}\right)^{\circ}$ and a closed set is a regular closed set if $Y=\left(Y^{\circ}\right)^{\downarrow}$. The complement of a regular open set is a regular closed set.

\subsection{Cubical complexes}

Let $\mathbb{Z}$ be the set of integers. Let $\mathbb{F}_{0}^{1}=\{\{a\} \mid a \in \mathbb{Z}\}$ and $\mathbb{F}_{1}^{1}=\{\{a, a+1\} \mid a \in \mathbb{Z}\}$. Let $n \geq 1$.

Let $f \subset \mathbb{Z}^{n}$. If $f$ is the Cartesian product of $m$ elements of $\mathbb{F}_{1}^{1}$ and $n-m$ elements of $\mathbb{F}_{0}^{1}$, we say that $f$ is a face or an $m$-face (of $\mathbb{Z}^{n}$ ), $m$ is the dimension of $f$, and we write $\operatorname{dim}(f)=m$ (some faces of $\mathbb{Z}^{2}$ are depicted in Figure 1). We denote by $\mathbb{F}^{n}$ the set composed of all faces of $\mathbb{Z}^{n}$; this set is the (n-D) space of cubical complexes. We denote by $\mathbb{F}_{k}^{n}$ $(0 \leq k \leq n)$ the set composed of all $k$-faces of $\mathbb{Z}^{n}$. The couple

${ }^{1}$ For the sake of readability, a discrete interval will be noted $\llbracket a, b \rrbracket$, while a continuous one will be noted $[a, b]$.

2 In a poset, this definition is equivalent to the classical one for which a set is connected if it cannot be split in two open (or closed) non empty sets.
$\left(\mathbb{F}^{n}, \subseteq\right)$ is a poset. Let $F \subseteq \mathbb{F}^{n}$ be a set of faces ${ }^{3}$. Let $f \in F$ be a face. The face $f$ is a facet of $F$ if $f$ is maximal in $F$. In particular, if $x=\left(x_{i}\right)_{i=1}^{n} \in \mathbb{Z}^{n}$, the set $\dot{x}=\prod_{i=1}^{n}\left\{x_{i}, x_{i}+1\right\}$ is a facet of $\mathbb{F}^{n}$.

Some technical lemmas, essentially devoted to cubical complexes, and needed in the sequel are provided in Appendix A.

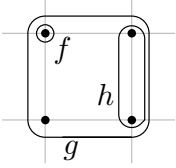

(a)

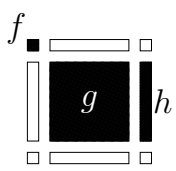

(b)
Fig. 1 Two representations of a set of faces $F=\{f, g, h\}$ in $\mathbb{Z}^{2}$ with, for instance, $f=\{0\} \times\{1\}, g=\{0,1\} \times\{0,1\}$ and $h=\{1\} \times\{0,1\}$. The face $g$ is a facet of $F$ (and also of $\mathbb{F}^{2}$ ).

\subsection{Digital topology}

Let $x=\left(x_{i}\right)_{i=1}^{n}$ and $y=\left(y_{i}\right)_{i=1}^{n}$ be two distinct points in $\mathbb{Z}^{n}$ (also called $n$-xels, or simply xels). The points $x$ and $y$ are $2 n$-adjacent if $\sum_{i=1}^{n}\left|x_{i}-y_{i}\right|=1$. They are $\left(3^{n}-1\right)$-adjacent if $\max _{i=1}^{n}\left\{\left|x_{i}-y_{i}\right|\right\}=1$. When $n=3$, the points $x$ and $y$ are 18-adjacent if they are 26-adjacent and $\sum_{i=1}^{n}\left|x_{i}-y_{i}\right| \leq 2$. Let $\alpha \in\left\{2 n, 3^{n}-1\right\}$ (or possibly $\alpha=18$ if $n=3$ ). Any point in $\mathbb{Z}^{n}$ is $\alpha$-adjacent to $\alpha$ other points. A sequence $\gamma=\left(z_{i}\right)_{i=0}^{r}$ ( $r \geq 0$ ) of points in $X \subseteq \mathbb{Z}^{n}$ is a (digital) $\alpha$-path ${ }^{4}$ (from $z_{0}$ to $z_{r}$ ) if for all $i \in \llbracket 1, r \rrbracket, z_{i-1}$ and $z_{i}$ are $\alpha$-adjacent. The integer $r$ is the length of $\gamma$. A subset $X \subseteq \mathbb{Z}^{n}$ is $\alpha$-connected, if for all $x, y \in X$, there exists a digital $\alpha$-path from $x$ to $y$ in $X$. In order to retrieve some topological features in binary digital images (such as the notion of hole), it is necessary to use pairs of adjacencies, one for the object $X$ and one for the background $\mathbb{Z}^{n} \backslash X$. The suitable pairs are $\left(2 n, 3^{n}-1\right)$ and $\left(3^{n}-1,2 n\right)$ (plus, when $n=3,(6,18)$ and $\left.(18,6)\right)$.

\section{Connectivity: from $\mathbb{Z}^{n}$ to $\mathbb{T}^{n}$}

A (digital) image $\lambda$ on $\mathbb{Z}^{n}$ is a function from $\mathbb{Z}^{n}$ to $\{0,1\}$. A (complex) image $\mu$ on $\mathbb{F}^{n}$ is a function from $\mathbb{F}^{n}$ to $\{0,1\}$. The object (resp. the background) associated to the image $\theta: X \rightarrow\{0,1\}$ (with $X=\mathbb{Z}^{n}$ or $\mathbb{F}^{n}$ ) is the set $\theta^{-1}(\{1\})$ (resp. $\left.\theta^{-1}(\{0\})\right)$.

\footnotetext{
${ }^{3}$ In the literature devoted to cubical complexes, it is generally assumed that $F \subseteq \mathbb{F}^{n}$ is a finite set of faces. This constraint can however be relaxed in the context of this work.

${ }^{4}$ We keep the usual designation though it would be more coherent in this work to define such a sequence of points as an $\alpha$-arc in order to reserve the word path for the functional point of view.
} 
If $\mu$ is a complex image, then we write $\bigvee_{x \in X} \mu(x)$ (resp. $\bigwedge_{x \in X} \mu(x)$ ) for the maximum (resp. minimum) of the set $\{\mu(x) \mid x \in X\}$ and we also write $\mu(a) \vee \mu(b)($ resp. $\mu(a) \wedge \mu(b))$ for $\bigvee_{x \in\{a, b\}} \mu(x)$ (resp. $\left.\bigwedge_{x \in\{a, b\}} \mu(x)\right)$.

The poset $\left(\mathbb{F}^{n}, \subseteq\right)$ is equipped with its Alexandroff topology.

All along the article, we provide intuitive representations of (abstract) objects of $\mathbb{F}^{n}$ by using different boxes for faces of different dimensions. Furthermore, in $\mathbb{F}^{2}$, the faces of the object are depicted in black while the faces of the background are in white, or are not shown, and, in $\mathbb{F}^{3}$, we use different colours for the faces of the object (blue: 3-faces; green: 2-faces; yellow: 1-faces; red: 0-faces) while the background is not represented.

\subsection{One-to-one correspondence between images on $\mathbb{Z}^{n}$ and $\mathbb{F}^{n}$}

When two faces $g, h \in \mathbb{F}^{n}$ cover a face $f \in \mathbb{F}^{n}$ and their smallest neighbourhoods do not intersect (i.e., $g^{\uparrow} \cap h^{\uparrow}=\emptyset$ ), we say that their are opposite with respect to the face $f$ (see Figure 2). We denote opp $(f)$ the set of all $\{g, h\}$ for $g$ opposite to $h$ w.r.t. $f$. Intuitively, the face $f$ is required to "locally connect" the faces $g$ and $h$. When $f$ is a facet, we have $\operatorname{opp}(f)=\emptyset$.

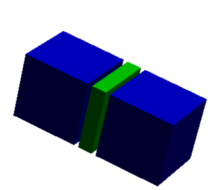

(a)

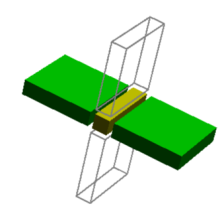

(b)

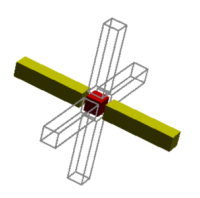

(c)
Fig. 2 Two opposite faces in $\mathbb{F}^{3}$ with respect to (a) a 2-face, (b) a 1face, (c) a 0 -face. The dashed boxes in (b) and (c) show other pairs of opposite faces.

Definition 1 (regular image) Let $\varepsilon: \llbracket 1, n \rrbracket \rightarrow\{-1,1\}$ be a function called connectivity function ${ }^{5}$. A function $\mu: \mathbb{F}^{n} \rightarrow$ $\{0,1\}$ is an $\varepsilon$-regular image (or simply a regular image) if for all $m \in \llbracket 1, n \rrbracket$ and $f \in \mathbb{F}_{m-1}^{n}$, we have, recursively

$\mu(f)= \begin{cases}\bigwedge_{\{a, b\} \in \operatorname{opp}(f)} \mu(a) \vee \mu(b) & \text { if } \varepsilon(m)=1 \\ \bigvee_{\{a, b\} \in \operatorname{opp}(f)} \mu(a) \wedge \mu(b) & \text { if } \varepsilon(m)=-1\end{cases}$

Examples of regular images are depicted in Figure 3.

\footnotetext{
5 By abuse of notation, we will often write a connectivity function $\varepsilon: \llbracket 1, n \rrbracket \rightarrow\{-1,1\}$ by exhaustively providing its values, i.e., by denoting $(\varepsilon(i))_{i=1}^{n}$ instead of $\varepsilon$, as done for instance in Figure 4(b,c). We will also use a dot symbol (.) when an $\varepsilon(i)$ value does not influence the behaviour of the $\varepsilon$ function on a given object, as done for instance in Figure 5(a). For the sake of simplicity, we will also write $\varepsilon=1$ (or $\varepsilon=-1$ ) when $\varepsilon$ is a constant function, as done for instance in Figure $4(\mathrm{a}, \mathrm{d})$.
}

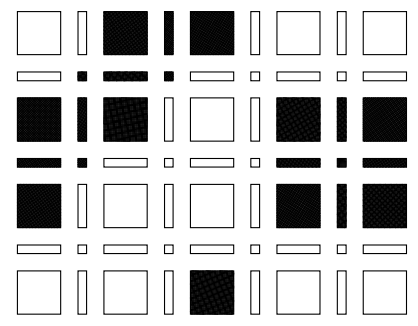

(a)

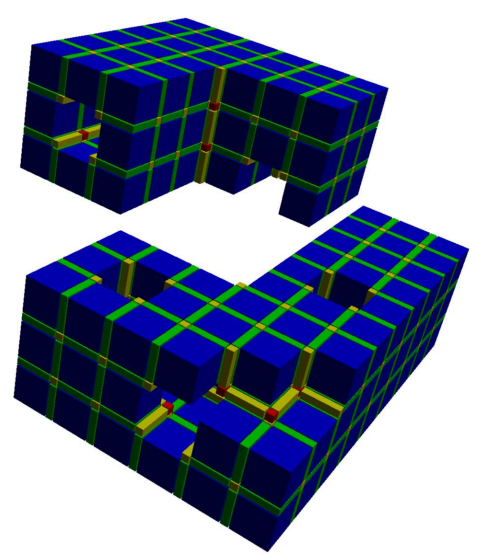

(b)

Fig. 3 Two $\varepsilon$-regular images (a) on $\mathbb{F}^{2}$ with $\varepsilon(2)=-1, \varepsilon(1)=1$, (b) on $\mathbb{F}^{3}$ with $\varepsilon(3)=-1, \varepsilon(2)=\varepsilon(1)=1$.

For each connectivity function $\varepsilon: \llbracket 1, n \rrbracket \rightarrow\{-1,1\}$, we define the function $\zeta_{\varepsilon}:\{0,1\}^{\mathbb{Z}^{n}} \rightarrow\{0,1\}^{\mathbb{F}^{n}}$ which maps any digital image $\lambda$ to the unique $\varepsilon$-regular image $\zeta_{\varepsilon}(\lambda)$ such that, for each $a \in \mathbb{Z}^{n}$, we have $\zeta_{\varepsilon}(\lambda)(\dot{a})=\lambda(a)$. It is obvious that, for each $\varepsilon$, the function $\zeta_{\varepsilon}$ is a bijection between the set of digital images $\{0,1\}^{Z^{n}}$ and the subset of $\varepsilon$-regular images of $\{0,1\}^{\mathbb{F}^{n}}$. Moreover, thanks to the choice of the connectivity function $\varepsilon$, we can accurately "carve" an image in $\mathbb{F}^{n}$ to model the desired connectivity in $\mathbb{Z}^{n}$ (see Figure 4). In particular, we can get the usual pairs of adjacencies ${ }^{6}$ (see Figures 4-7 and Table 1). In Section 4, the correspondences given in Table 1 will be justified by two theorems establishing that, by following these links, we preserve the connected components and fundamental groups.

When the function $\varepsilon$ is constant, Definition 1 can be simplified. Note that the case $\varepsilon=-1$ corresponds to the $2 n$ adjacency in $\mathbb{Z}^{n}$ while the case $\varepsilon=1$ corresponds to the $\left(3^{n}-1\right)$-adjacency in $\mathbb{Z}^{n}$.

Proposition 1 Let $\mu: \mathbb{F}^{n} \rightarrow\{0,1\}$ be an $\varepsilon$-regular image. Let $f$ be a face of $\mathbb{F}^{n}$.

\footnotetext{
${ }^{6}$ Note that each one of the $2^{n}$ connectivity functions $\varepsilon: \llbracket 1, n \rrbracket \rightarrow$ $\{-1,1\}$ may enable to retrieve a standard adjacency for the object (possibly redundantly, see Figure 5(c,d)), but does not necessarily induces a standard pair of adjacencies, as exemplified in Figures 6 and 7.
} 


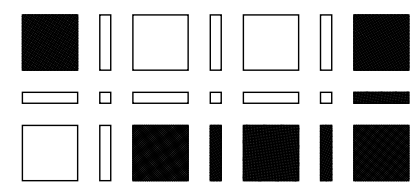

(a) $\varepsilon=-1$

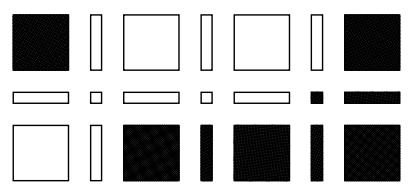

(b) $\varepsilon=(1,-1)$

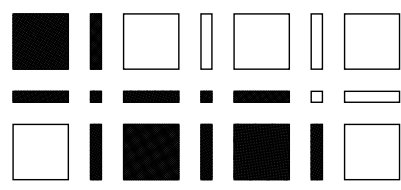

(c) $\varepsilon=(-1,1)$

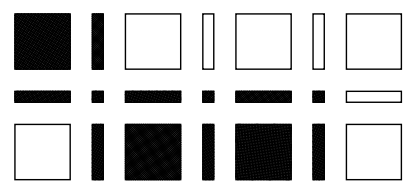

(d) $\varepsilon=1$

Fig. 4 Images $\zeta_{\varepsilon}(\lambda): \mathbb{F}^{2} \rightarrow\{0,1\}$ for some given $\lambda: \mathbb{Z}^{2} \rightarrow\{0,1\}$.

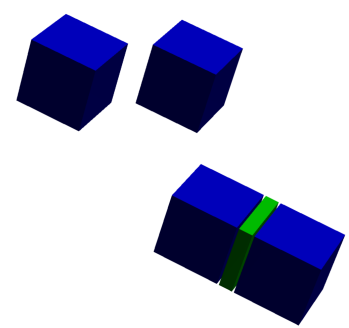

(a) $\varepsilon=(., .,-1)$

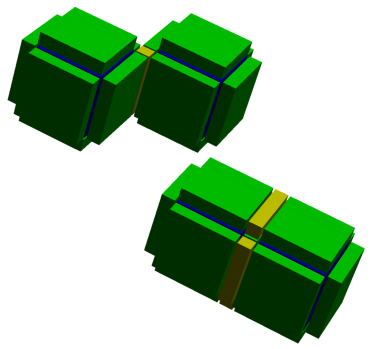

(b) $\varepsilon(3)=1, \varepsilon(2)=-1$

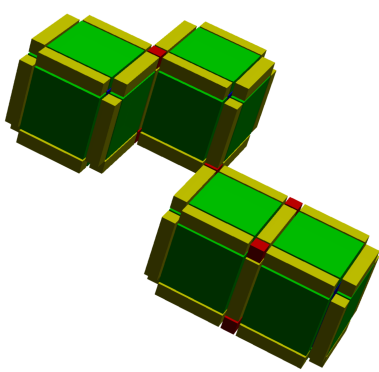

(c) $\varepsilon(3)=\varepsilon(2)=1, \varepsilon(1)=-1$

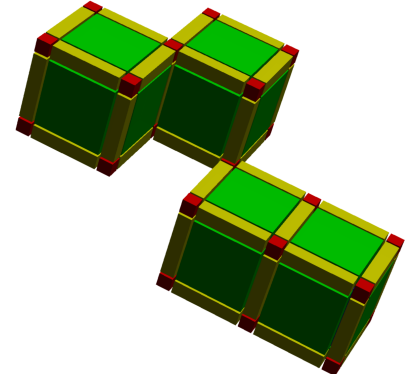

(d) $\varepsilon=1$

Fig. 5 Images $\zeta_{\varepsilon}(\lambda): \mathbb{F}^{3} \rightarrow\{0,1\}$ for some given $\lambda: \mathbb{Z}^{3} \rightarrow\{0,1\}$. (a) With $\varepsilon(3)=-1$, we obtain the 6-adjacency in $\mathbb{Z}^{3}$. (b) With $\varepsilon(3)=1$ and $\varepsilon(2)=-1$, we obtain the 18 -adjacency in $\mathbb{Z}^{3}$. (c, d) With $\varepsilon(3)=\varepsilon(2)=1$, we obtain the 26-adjacency in $\mathbb{Z}^{3}$.

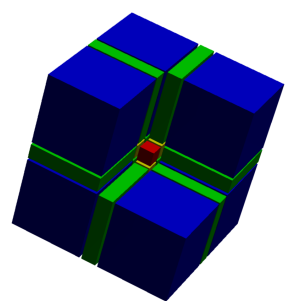

(a) $\varepsilon(3)=\varepsilon(2)=-1, \varepsilon(1)=1$

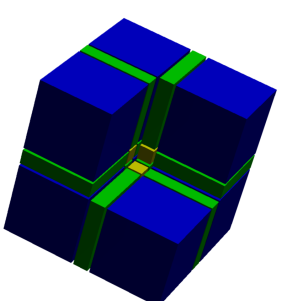

(b) $\varepsilon(3)=\varepsilon(2)=\varepsilon(1)=-1$

Fig. 6 The (subtle) difference between the connectivity functions $(1,-1,-1)$ and $(-1,-1,-1)$.

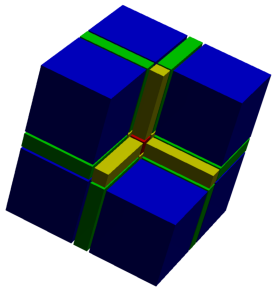

(a) $\varepsilon(3)=-1, \varepsilon(2)=1$

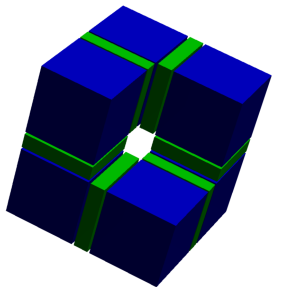

(b) $\varepsilon(3)=\varepsilon(2)=-1$

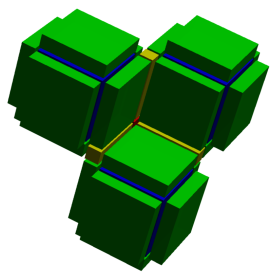

(c) $\varepsilon(3)=\varepsilon(1)=1, \varepsilon(2)=-1$

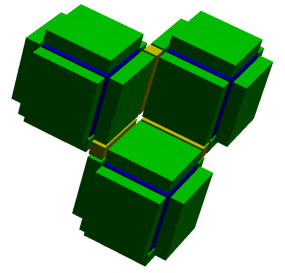

(d) $\varepsilon(3)=1, \varepsilon(2)=\varepsilon(1)=-1$

Fig. 7 (a,b) A torus built with six 3-faces illustrates how the two 6-adjacencies can be obtained. There are two 3-faces of the background which intersect the six 3-faces of the torus. These two white-faces are 26-adjacent but in (a) they do not intersect in the background (for the torus is a horn-torus) while, in (b), they do (the torus is a ring-torus). Hence, in (a), we model a $(6,18)$-adjacency relation while in $(b)$, we model a $(6,26)$ adjacency relation. (c,d) An object built from three facets with two connectivity functions which could a priori be used to model the 18-adjacency (see Figure 5(b)). In (c) we can see a red 0-face between the three cubes. This is what is expected for the background must have a 6-adjacency. In (d), there is a hole instead of the red 0 -face, which is not correct in 18-adjacency. 


\begin{tabular}{|c|c|c|c|c|c|c|c|c|}
\hline Space dimension & \multicolumn{2}{|c|}{$n=2$} & \multicolumn{4}{|c|}{$n=3$} & \multicolumn{2}{|c|}{$n \geq 4\left(\right.$ actually, $\left.n \in \mathbb{N}^{*}\right)$} \\
\hline Adjacencies in $\mathbb{Z}^{n}$ & $(4,8)$ & $(8,4)$ & $(6,26)$ & $(6,18)$ & $(18,6)$ & $(26,6)$ & $\left(2 n, 3^{n}-1\right)$ & $\left(3^{n}-1,2 n\right)$ \\
\hline $\begin{array}{l}\text { Connectivity } \\
\text { function }\end{array}$ & $\varepsilon=-1$ & $\varepsilon=1$ & $\varepsilon=-1$ & $\begin{array}{l}\varepsilon(3)=-1 \\
\varepsilon(2)=1 \\
\varepsilon(1)=-1\end{array}$ & $\begin{array}{l}\varepsilon(3)=1 \\
\varepsilon(2)=-1 \\
\varepsilon(1)=1\end{array}$ & $\varepsilon=1$ & $\varepsilon=-1$ & $\varepsilon=1$ \\
\hline
\end{tabular}

Table 1 Correspondence between pairs of adjacencies in $\mathbb{Z}^{n}$ and connectivity functions.

(i) If $\forall m>\operatorname{dim}(f), \varepsilon(m)=-1$, then we have

$$
\mu(f)=\bigwedge_{f<a} \mu(a)=\bigwedge_{a \in f^{\uparrow+}} \mu(a)
$$

(ii) If $\forall m>\operatorname{dim}(f), \varepsilon(m)=1$, then we have

$$
\mu(f)=\bigvee_{f<a} \mu(a)=\bigvee_{a \in f^{\uparrow+}} \mu(a)
$$

In particular, if $\varepsilon=-1$ (resp. $\varepsilon=1)$ then $\mu(f)=\bigwedge_{a \in f^{\uparrow+}} \mu(a)$ (resp. $\left.\mu(f)=\bigvee_{a \in f^{\uparrow+}} \mu(a)\right)$ for all $f \in \mathbb{F}^{n}$.

Proof The proof is restricted to part (i), since part (ii) can be easily obtained by duality. We build the proof by induction on $k=n-\operatorname{dim}(f) \geq 1$. When $k=1$, there are two facets $g, h$ such that $f^{\uparrow+}=f^{<}=\{g, h\}$ and opp $(f)=\{\{g, h\}\}$. Thus, since $\varepsilon(n)=-1$, we have $\mu(f)=\bigvee_{\{a, b\} \in \operatorname{opp}(f)} \mu(a) \wedge \mu(b)=$ $\mu(g) \wedge \mu(h)=\bigwedge_{a \in f^{\uparrow+}} \mu(a)=\bigwedge_{f<a} \mu(a)$. Suppose now that, for some $k \in \llbracket 1, n-1 \rrbracket, \forall m \geq n-k, \varepsilon(m)=-1$ and for each face $b$ of dimension $n-k, \mu(b)=\bigwedge_{b<a} \mu(a)=\bigwedge_{a \in f^{\uparrow+}} \mu(a)$. Let $f$ be a face of dimension $n-k-1$. From Definition 1 , we have $\mu(f)=\bigvee_{\{a, b\} \in \operatorname{opp}(f)} \mu(a) \wedge \mu(b)$, and from the induction hypothesis, it then comes

$\left.\mu(f)=\bigvee_{\{a, b\} \in \operatorname{opp}(f)}\left(\bigwedge_{c \in a^{\uparrow+}} \mu(c)\right) \wedge\left(\bigwedge_{c \in b^{\uparrow+}} \mu(c)\right)\right)$

and thus

$\mu(f)=\bigvee_{\{a, b\} \in \operatorname{opp}(f)} \bigwedge_{c \in a^{\uparrow+\cup b^{\uparrow+}}} \mu(c)$

From Lemma 7(ii) (see Appendix A), we have $f^{\uparrow+}=a^{\uparrow+} \cup$ $b^{\uparrow+}$ for all $(a, b) \in \operatorname{opp}(f)$. Hence, we obtain :

$\mu(f)=\bigvee_{\{a, b\} \in \operatorname{opp}(f)} \bigwedge_{c \in f^{\uparrow+}} \mu(c)=\bigwedge_{c \in f^{\uparrow+}} \mu(c)$

Using again the induction hypothesis, it finally comes

$\bigwedge_{f<a} \mu(a)=\bigwedge_{f<a} \bigwedge_{b \in a^{\uparrow+}} \mu(b)=\bigwedge_{b \in f^{\uparrow+}} \mu(b)=\mu(f)$

Hence, the result holds.

\subsection{Duality}

Let $\theta: X \rightarrow\{0,1\}$ with $\left(X=\mathbb{Z}^{n}\right.$ or $\left.\mathbb{F}^{n}\right)$ be an image. We define the negative image $\neg \theta: X \rightarrow\{0,1\}$ of $\theta$ by $\neg \theta(x)=$ $\neg(\theta(x))=1-\theta(x)$, for all $x \in X$.

In the following, $\varepsilon$ denotes an arbitrary connectivity function.

Proposition 2 Let $\mu: \mathbb{F}^{n} \rightarrow\{0,1\}$ be an $\varepsilon$-regular image. Then $\neg \mu$ is a $(-\varepsilon)$-regular image.

Proof Let $f \in \mathbb{F}_{m-1}^{n}$, with $1 \leq m \leq n$. Let us suppose that $\varepsilon(m)=1$. Then, $(\neg \mu)(f)=\neg(\mu(f))=\neg\left(\bigwedge_{\{a, b\} \in \mathrm{opp}(f)} \mu(a) \vee\right.$ $\mu(b))$. From De Morgan's law, we infer:

$$
\begin{aligned}
(\neg \mu)(f) & =\bigvee_{(a, b) \in \operatorname{opp}(f)} \neg(\mu(a)) \wedge \neg(\mu(b)) \\
& =\bigvee_{(a, b) \in \operatorname{opp}(f)}(\neg \mu)(a) \wedge(\neg \mu)(b) .
\end{aligned}
$$

The case $\varepsilon(m)=-1$ is similar. Whence, $\neg \mu$ is $(-\varepsilon)$-regular.

Let $\mu: \mathbb{F}^{n} \rightarrow\{0,1\}$ be an $\varepsilon$-regular image. We define the image $-\mu: \mathbb{F}^{n} \rightarrow\{0,1\}$ by $(-\mu)(f)=\mu(f)$ for all $f \in \mathbb{F}_{n}^{n}$ and $\mu$ is $(-\varepsilon)$-regular, as exemplified in Figure 8.

Proposition 3 Let $\mu: \mathbb{F}^{n} \rightarrow\{0,1\}$ be an $\varepsilon$-regular image. Then we have $\neg(-\mu)=-(\neg \mu)$.

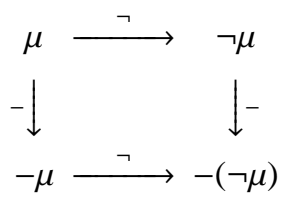

Proof Let $f \in \mathbb{F}_{n}^{n}$. We have $(\neg(-\mu))(f)=\neg((-\mu)(f))=$ $\neg(\mu(f))=(\neg \mu)(f)=(-(\neg \mu))(f)$. Furthermore, $-\mu$ and $\neg \mu$ are $(-\varepsilon)$-regular. So, $\neg(-\mu)$ and $-(\neg \mu)$ are $\varepsilon$-regular. Thus, $\neg(-\mu)=-(\neg \mu)$.

From the above definitions and propositions, we straightforwardly derive the following result.

Proposition 4 Let $\lambda: \mathbb{Z}^{n} \rightarrow\{0,1\}$ be a digital image. Let $\varepsilon: \llbracket 1, n \rrbracket \rightarrow\{-1,1\}$ be a connectivity function. Then, we have $\neg\left(\zeta_{\varepsilon}(\lambda)\right)=\zeta_{-\varepsilon}(\neg \lambda)$.

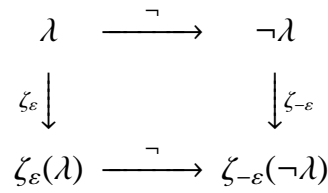

Propositions 3 and 4 are illustrated in Figures 8 and 9. 


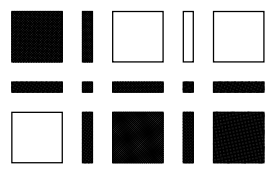

(a) $\mu$

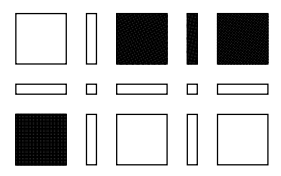

(c) $\neg \mu$

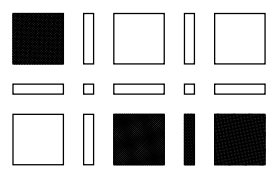

(b) $-\mu$

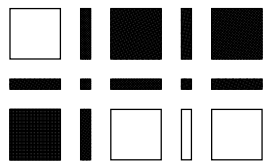

(d) $-(\neg \mu)=\neg(-\mu)$
Fig. 8 (a) A $(-1,1)$-regular image $\mu: \mathbb{F}^{2} \rightarrow\{0,1\}$. (b) The $(1,-1)$ regular image $-\mu$. (c) The $(1,-1)$-regular image $\neg \mu$. (d) The $(-1,1)$ regular image $-(\neg \mu)=\neg(-\mu)$.

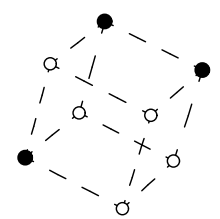

(a) $\lambda$

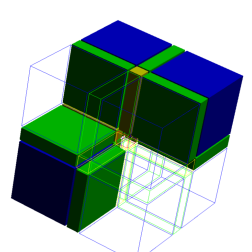

(c) $\zeta_{\varepsilon}(\lambda)$

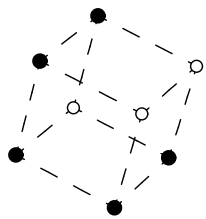

(b) $\neg \lambda$ (rotated)

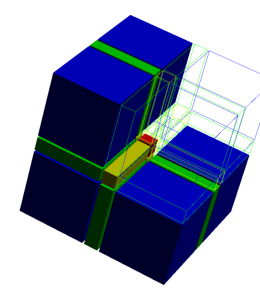

(d) $\zeta_{-\varepsilon}(\neg \lambda)=\neg\left(\zeta_{\varepsilon}(\lambda)\right)$
Fig. 9 (a) A digital image $\lambda: \mathbb{Z}^{2} \rightarrow\{0,1\}$. (b) The negative image $\neg \lambda$ associated to $\lambda$. (c) The $\varepsilon$-regular image $\zeta_{\varepsilon}(\lambda)$ associated to $\lambda$ with $\varepsilon=(1,-1,-1)$. (d) The $(-\varepsilon)$-regular image $\zeta_{-\varepsilon}(\neg \lambda)$ associated to $\neg \lambda$. Note that $(b, d)$ have been rotated of $-\pi / 2$ along the (nearly) vertical axis in order to ease the visualisation of (d).

Remark 1 This proposition establishes that, for a given connectivity function $\varepsilon$ (and the associated pair of adjacencies $(\alpha, \beta))$, all the properties valid for $\lambda^{-1}(\{1\})$ and $\mu^{-1}(\{1\})$ are also valid for $\lambda^{-1}(\{0\})$ and $\mu^{-1}(\{0\})$ for the opposite connectivity function $-\varepsilon$ (and the associated pair of adjacencies $(\beta, \alpha)$ ). Broadly speaking, this means that the notions of object and background can be switched without loss of generality, provided that the pair of adjacencies $(\beta, \alpha)$ is also switched accordingly.

\subsection{Image values and connectivity functions}

The values of the facets of an $\varepsilon$-regular image on $\mathbb{F}^{n}$ are directly provided by its associated digital image on $\mathbb{Z}^{n}$. How- ever, the values of the lower-dimensional faces depend on the connectivity function $\varepsilon$. In this section, we state some general properties related to links between these values and the considered connectivity functions.

The following lemma is a straightforward consequence of Definition 1.

Lemma 1 Let $\mu: \mathbb{F}^{n} \rightarrow\{0,1\}$ be an $\varepsilon$-regular image. Let $f \in \mu^{-1}(\{1\})$ be such that $\operatorname{dim}(f)<n$. Then, either $\varepsilon(\operatorname{dim}(f)+$ 1) $=-1$ and there exists $\{a, b\} \in \operatorname{opp}(f)$ such that $\mu(a)=$ $\mu(b)=1$, or $\varepsilon(\operatorname{dim}(f)+1)=1$ and for all $\{a, b\} \in \operatorname{opp}(f)$, $\mu(a)=1$ or $\mu(b)=1$.

By applying $n-\operatorname{dim}(f)$ times Lemma 1 , we deduce the following proposition.

Proposition 5 Let $\mu: \mathbb{F}^{n} \rightarrow\{0,1\}$ be an $\varepsilon$-regular image. Let $f \in \mu^{-1}(\{1\})$. Then, for each $k \in \llbracket \operatorname{dim}(f)+1, n \rrbracket$ there exists a face $g \in f^{\uparrow} \cap \mathbb{F}_{k}^{n}$ such that $\mu(g)=1$.

The next proposition -easy to prove by induction- ensures that in a region of facets of uniform values, the image has a constant value in all dimensions.

Proposition 6 Let $\mu: \mathbb{F}^{n} \rightarrow\{0,1\}$ be a regular image. Let $x \in\{0,1\}$ and $f \in \mathbb{F}^{n}$. If, for each facet $g$ in $f^{\uparrow+}, \mu(g)=x$, then $\mu(f)=x$.

The following proposition establishes a partial converse of Proposition 1. In the sequel, we write $f_{1}^{\uparrow+}$ for the set of facets in the star of $f$ which have value $1: f_{1}^{\uparrow+}=\left\{g \in \mathbb{F}_{n}^{n} \mid\right.$ $f \leqslant g$ and $\mu(g)=1\}$.

Proposition 7 Let $\mu: \mathbb{F}^{n} \rightarrow\{0,1\}$ be an $\varepsilon$-regular image. Let $f \in \mu^{-1}(\{1\})$. If there exists a unique facet in $f_{1}^{\uparrow+}$, then $\varepsilon(k)=1$ for all $k \in \llbracket \operatorname{dim}(f)+1, n \rrbracket$.

Proof The proof is made par induction on $n-\operatorname{dim}(f)$. For $\operatorname{dim}(f)=n$, the property is obvious. Let us now suppose that the property is true for any face of dimension greater or equal to a given $m \in \llbracket 1, n \rrbracket$. Let $f \in \mathbb{F}_{m-1}^{n}$ be such that $f \in \mu^{-1}(\{1\})$ and there exists a unique facet $g$ in $f_{1}^{\uparrow+}$. Let $h$ be a face in $f^{<}$. If $h \in f^{<} \backslash g^{\downarrow}$ (and such a face exists), none of the facets in $h^{\uparrow}$ are in $\mu^{-1}(\{1\})$. Thus, from Proposition 5, we derive that $h \notin \mu^{-1}(\{1\})$ and, by Lemma 1 , we know that not all the faces $h$ in $f^{<}$can verify $h \notin \mu^{-1}(\{1\})$. Hence, there exists $h \in f^{<} \cap g^{\downarrow}$ such that $h \in \mu^{-1}(\{1\})$ and, from the induction hypothesis, we deduce that $\varepsilon(k)=1$ for all $k \geq m+1$. Let $(a, b) \in \operatorname{opp}(f)$. From Lemma 7(ii), we derive that $a \notin g^{\downarrow}$ or $b \notin g^{\downarrow}$. Therefore, $\mu(a)=0$ or $\mu(b)=0$ and $\mu(a) \wedge \mu(b)=0$. Since this last equality is true for each pair in opp $(f)$, we can state that $\bigvee_{\{a, b\} \in \operatorname{opp}(f)} \mu(a) \wedge \mu(b)=0$ So, $\varepsilon(m)$ cannot be equal to -1 . Hence, $\varepsilon(m)=1$, and then $\varepsilon(k)=1$ for all $k \geq m$.

Hereafter, we study the value of an intersection of facets. This is an important issue, which will especially be considered in Section 4. 
Proposition 8 Let $\mu: \mathbb{F}^{n} \rightarrow\{0,1\}$ be an $\varepsilon$-regular image. Let $f, g$ be two distinct facets of $\mu^{-1}(\{1\})$ with $f \cap g \neq \emptyset$. If $\varepsilon(k)=1$ for all $k \in \llbracket \operatorname{dim}(f \cap g)+2, n \rrbracket$, then $\mu(f \cap g)=1$. Conversely, if $\mu(f \cap g)=1$ and the only facets in $(f \cap g)_{1}^{\uparrow+}$ are $f$ and $g$, then $\varepsilon(k)=1$ for all $k \in \llbracket \operatorname{dim}(f \cap g)+2, n \rrbracket$.

Proof We set $h=f \cap g$ and $d=\operatorname{dim}(h)+1$. Since $\mu(f)=$ $\mu(g)=1$ and $\mu(a)=\bigvee_{b \in a^{\uparrow+}} \mu(b)$ for each face $a$ such that $\operatorname{dim}(a) \in \llbracket d, n-1 \rrbracket$ (Proposition 1), all the $k$-faces included in $f$ or $g$, with $k \geq d$, are in $\mu^{-1}(\{1\})$. In particular, thanks to Lemma 6, we derive $h^{<} \subseteq \mu^{-1}(\{1\})$ and, therefore, whatever is the value of $\varepsilon(d), h \in \mu^{-1}(\{1\})$. Conversely, any face $a \in$ $h^{<}$is either included in $f$ or in $g$ (Lemma 6), but not in both of them. Since the only facets in $(f \cap g)_{1}^{\uparrow+}$ are $f$ and $g$, we can use Proposition 7 to conclude that $\varepsilon(k)=1$ for all $k \geq d+1$.

Proposition 9 Let $\mu: \mathbb{F}^{n} \rightarrow\{0,1\}$ be an $\varepsilon$-regular image. Let $f, g$ be two distinct facets of $\mu^{-1}(\{1\})$ with $f \cap g \neq \emptyset$. If $\mu(f \cap g)=1$, then there exists an arc $q=\left(q_{i}\right)_{i=0}^{2 r}(r \geq 0)$ from $f$ to $g$ in $(f \cap g)^{\uparrow} \cap \mu^{-1}(\{1\})$ such that, for all $i \in \llbracket 0, r \rrbracket$, $q_{2 i} \in \mathbb{F}_{n}^{n}$ and for all $i \in \llbracket 0, r-1 \rrbracket, q_{2 i+1}=q_{2 i} \cap q_{2(i+1)} \in \mathbb{F}_{m}^{n}$ with $m \in \llbracket k, n-1 \rrbracket$ where $k \in \llbracket 0, n-1 \rrbracket$ is the lowest integer such that $\varepsilon(i)=1$ for all $i \in \llbracket k+2, n \rrbracket$.

Proof Let $\mu$ be an $\varepsilon$-regular image and $k \in \llbracket 0, n-1 \rrbracket$ be an integer such that $\varepsilon(i)=1$ for all $i \in \llbracket k+2, n \rrbracket$ and $\varepsilon(k+1)=$ -1 (see Figure 10 for examples). Let $f, g$ be two facets of $\mu^{-1}(\{1\})$ such that $f \cap g \neq \emptyset$ and $\mu(f \cap g)=1$. The proof is carried out by induction on $k-\operatorname{dim}(f \cap g)$. If $k \leq \operatorname{dim}(f \cap g)$, the property is obvious since we can set $q=(f, f \cap g, g)$. We suppose now that the property is true for any facets $i, j$ such that $\operatorname{dim}(i \cap j) \geq k-m$ for some $m \in \llbracket 0, k-1 \rrbracket$. Let $f, g$ be such that $\operatorname{dim}(f \cap g)=k-m-1$. There exists a face $a$ such that $f \cap g<a$ and $\mu(a)=1$ (Lemma 1) and $a$ is included in $f$ or in $g$ (Lemma 6) but not in both of them. Since, $\varepsilon(k+1)=-1$ and $k+1 \geq \operatorname{dim}(a)+1$, we derive from Proposition 7 that there exists a facet $h, h \neq f$ and $h \neq g$, in $a^{\uparrow} \cap \mu^{-1}(\{1\})$. It is easy to see that as $f, g, h$ are three facets in $\mathbb{F}^{n}$ such that $f \cap g \subset h$, then $\operatorname{dim}(f \cap h)$ and $\operatorname{dim}(g \cap h)$ are strictly greater than $\operatorname{dim}(f \cap g)$ so we can use the induction hypothesis: there exist two arcs in $(f \cap g)^{\uparrow} \cap \mu^{-1}(\{1\}), q_{1}$ from $f$ to $h$ and $q_{2}$ from $h$ to $g$, whose faces of odd rank are facets of $\mathbb{F}^{n}$ and faces of even rank $i$ are the intersection of the facets of ranks $i-1$ and $i+1$ with a dimension belonging to $\llbracket k, n-1 \rrbracket$. Therefore, there exists an arc from $f$ to $g$ in $(f \cap g)^{\uparrow} \cap \mu^{-1}(\{1\})$ whose faces satisfy all the conditions of the proposition.

\subsection{Computing values directly from facets}

The aim of this section is to find the number of facets which must have the value 1 in the star of a face to ensure that this face also has value 1 . In $\mathbb{F}^{2}$, the answer is straightforward.

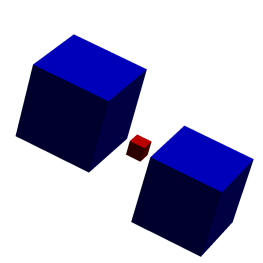

(a) $\varepsilon(3)=-1$

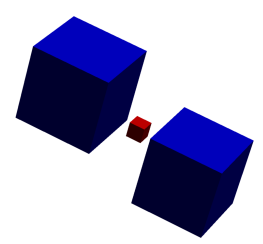

(c) $\varepsilon(3)=1, \varepsilon(2)=-1$

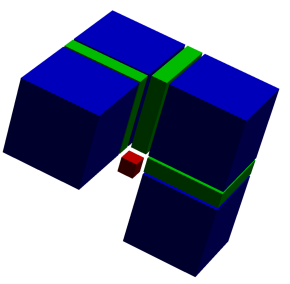

(b) $k=n-1=2$

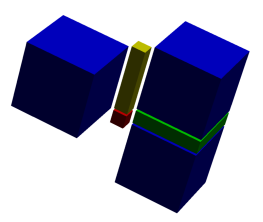

(d) $k=n-2=1$
Fig. 10 (a) If $\varepsilon(3)=-1$, the integer $k$ defined in Proposition 9 is equal to $n-1=2$. Then the proposition states that, since the intersection (in red) of the two facets of $\mu^{-1}(\{1\})$ (in blue) is in $\mu^{-1}(\{1\})$ there must exist a path composed of facets and of faces of dimension higher than 2 between these two facets, as the one depicted in (b). (c) If $\varepsilon(3)=$ $1, \varepsilon(2)=-1$, the integer $k$ defined in Proposition 9 is equal to $n-$ $2=1$. So, there must exist a path composed of facets and of faces of dimension higher than 1 between these two facets as the one depicted in (d).

In $\mathbb{F}^{3}$, it requires to carefully study a particular configuration (depicted in Figure 11), however, it can be answered, as stated hereafter. In higher dimensional spaces, the particular configurations to study are too numerous to get a useful result.

Let $f \in \mathbb{F}^{n}$, with $n \geq 3$. If $\operatorname{dim}(f)=n-3$, the poset $\left(f^{\uparrow}, \subseteq\right.$ ) has a unique minimum, namely $f$, and 8 maximal elements, namely the facets forming $f^{\uparrow+}$. From an adjacency point of view, these facets are geometrically organised as the 8 vertices of a cubical structure. When $f_{1}^{\uparrow+}$ (i.e., the facets of $f^{\uparrow+}$ whose values are equal to 1 ) is organised as in the configuration depicted in Figure 11(a) (up to rotations and symmetries), we say that $f_{1}^{\uparrow+}$ is a trihedron.

We define $\operatorname{Card}^{-}(E)=3$ and $\operatorname{Card}^{+}(E)=5$, if $E$ is a trihedron, and $\operatorname{Card}^{-}(E)=\operatorname{Card}^{+}(E)=\operatorname{Card}(E)$ otherwise.

For each connectivity function $\varepsilon$, we define recursively the function $\delta_{\varepsilon}$ by

$$
\begin{aligned}
\delta_{\varepsilon}: \llbracket 0, n \rrbracket & \rightarrow \llbracket 1,2^{n} \rrbracket \\
0 & \mapsto 1 \\
i+1 & \mapsto \begin{cases}2 \delta_{\varepsilon}(i)-1 & \text { if } \varepsilon(n-i)=1 \\
2 \delta_{\varepsilon}(i) & \text { if } \varepsilon(n-i)=-1\end{cases}
\end{aligned}
$$

It is easy to check that, for all $m \in \llbracket 0, n \rrbracket$, we have

$$
\delta_{\varepsilon}(m)=1+\sum_{k=1}^{m}(1-\varepsilon(n-k+1)) 2^{m-k-1}
$$

Proposition 10 Let $\mu: \mathbb{F}^{n} \rightarrow\{0,1\}$ be an $\varepsilon$-regular image. Let $f$ be a $k$-face of $\mathbb{F}^{n}$ (with $n-3 \leq k \leq n-1$ ). 


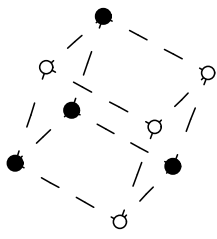

(a)

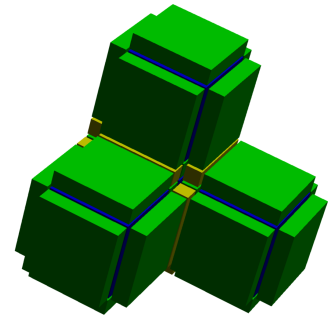

(b) $\varepsilon(3)=1, \varepsilon(2)=\varepsilon(1)=-1$

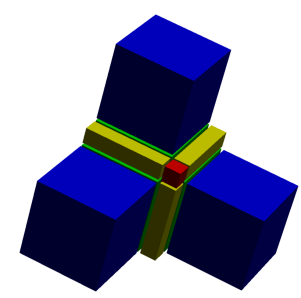

(c) $\varepsilon(3)=-1, \varepsilon(2)=\varepsilon(1)=1$

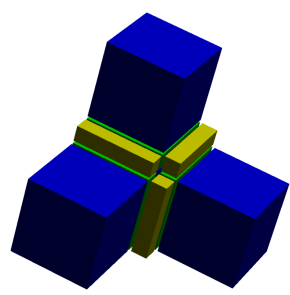

(d) $\varepsilon(3)=\varepsilon(1)=-1, \varepsilon(2)=1$

Fig. 11 (a) Symbolic representation of a trihedron related to a face $f \in \mathbb{F}^{n}$ such that $\operatorname{dim}(f)=n-3$. Black dots: $f_{1}^{\uparrow+}$; white dots $f^{\uparrow+} \backslash f_{1}^{\uparrow+}$. The dash lines represent the existence of a face of dimension $n-1$ forming the intersection between two facets of $f^{\uparrow+}$. (b-d) Examples of trihedra, with three connectivity functions $\varepsilon$ (one of the blue facets is hidden).

\begin{tabular}{|c|c|c|c|c|}
\hline \multicolumn{5}{|c|}{$\operatorname{dim}(f)=n-2$} \\
\hline$\varepsilon$ & $(\ldots, 1,1)$ & $(\ldots,-1,1)$ & $(\ldots, 1,-1)$ & $(\ldots,-1,-1)$ \\
\hline$C_{\varepsilon}$ & $\operatorname{Card}\left(f_{1}^{\uparrow+}\right) \geq 1$ & $\operatorname{Card}\left(f_{1}^{\uparrow+}\right) \geq 2$ & $\operatorname{Card}\left(f_{1}^{\uparrow+}\right) \geq 3$ & $\operatorname{Card}\left(f_{1}^{\uparrow+}\right) \geq 4$ \\
\hline
\end{tabular}

\begin{tabular}{|c|c|c|c|c|}
\hline \multicolumn{5}{|c|}{$\operatorname{dim}(f)=n-3$} \\
\hline$\varepsilon$ & $(\ldots, 1,1,1)$ & $(\ldots,-1,1,1)$ & $(\ldots, 1,-1,1)$ & $(\ldots,-1,-1,1)$ \\
\hline$C_{\varepsilon}$ & $\operatorname{Card}\left(f_{1}^{\uparrow+}\right) \geq 1$ & $\operatorname{Card}\left(f_{1}^{\uparrow+}\right) \geq 2$ & $\operatorname{Card}\left(f_{1}^{\uparrow+}\right) \geq 3$ & $\begin{array}{c}\operatorname{Card}\left(f_{1}^{\uparrow+}\right) \geq 4 \\
f_{1}^{\uparrow+} \operatorname{not} \text { a trihedron }\end{array}$ \\
\hline$\varepsilon$ & $(\ldots, 1,1,-1)$ & $(\ldots,-1,1,-1)$ & $(\ldots, 1,-1,-1)$ & $(\ldots,-1,-1,-1)$ \\
\hline$C_{\varepsilon}$ & $\begin{array}{c}\text { Card }\left(f_{1}^{\uparrow+}\right) \geq 5 \\
\text { or } f_{1}^{\uparrow+} \text { a trihedron }\end{array}$ & $\operatorname{Card}\left(f_{1}^{\uparrow+}\right) \geq 6$ & $\operatorname{Card}\left(f_{1}^{\uparrow+}\right) \geq 7$ & $\operatorname{Card}\left(f_{1}^{\uparrow+}\right)=8$ \\
\hline
\end{tabular}

Table 2 Necessary and sufficient conditions to obtain $\mu(f)=1$ (see Corollary 1 ).

(i) If $\operatorname{Card}^{-}\left(f_{1}^{\uparrow+}\right) \geq \delta_{\varepsilon}(n-k)$, then $\mu(f)=1$.

(ii) If $\mu(f)=1$, then $\operatorname{Card}^{+}\left(f_{1}^{\uparrow+}\right) \geq \delta_{\varepsilon}(n-k)$.

Proof We set $m=n-\operatorname{dim}(f)=n-k$ (note that $1 \leq m \leq 3)$. If $m=1$, then $\delta_{\varepsilon}(n-k)=\delta_{\varepsilon}(1)$ is equal to 1 if $\varepsilon(n)=1$ and to 2 if $\varepsilon(n)=-1$. Since, in the case $m=1, f^{\uparrow+}$ contains exactly two faces, which are opposite facets with respect to $f$, the statements (i) and (ii) are obviously true for $m=1$. Now let us suppose that $m \in \llbracket 2,3 \rrbracket$ and that the statements are true for $m-1$.

First case: $\varepsilon(\operatorname{dim}(f)+1)=-1$. If $\operatorname{Card}^{-}\left(f_{1}^{\uparrow+}\right) \geq \delta_{\varepsilon}(m)=$ $2 \delta_{\varepsilon}(m-1)$, then we deduce from Lemma 8 that there exist two faces $g$ and $h$,opposite with respect to $f$, such that $\operatorname{Card}\left(g_{1}^{\uparrow+}\right) \geq \delta_{\varepsilon}(m-1)$ and $\operatorname{Card}\left(h_{1}^{\uparrow+}\right) \geq \delta_{\varepsilon}(m-1)$. Since the dimension of the faces $g$ and $h$ is greater than or equal to $n-2$, we get $\operatorname{Card}\left(g_{1}^{\uparrow+}\right)=\operatorname{Card}^{-}\left(g_{1}^{\uparrow+}\right)$ and $\operatorname{Card}\left(h_{1}^{\uparrow+}\right)=$ $\operatorname{Card}^{-}\left(h_{1}^{\uparrow+}\right)$ so we can use the induction hypothesis to derive that $\mu(g)=\mu(h)=1$. Recalling that $\varepsilon(\operatorname{dim}(f)+1)=-1$, we have $\mu(f)=\bigvee_{\{a, b\} \in \operatorname{opp}(f)} \mu(a) \wedge \mu(b) \geq \mu(g) \wedge \mu(h)=1$, and thus $\mu(f)=1$. Then (i) holds. Conversely, if $\mu(f)=$ 1 , thanks to Lemma 1 we know that there exists $\{g, h\} \in$ $\operatorname{opp}(f)$ such that $\mu(g)=\mu(h)=1$. Hence, by the induction hypothesis, $\operatorname{Card}^{+}\left(g_{1}^{\uparrow+}\right)=\operatorname{Card}\left(g_{1}^{\uparrow+}\right) \geq \delta_{\varepsilon}(m-1)$ and $\operatorname{Card}^{+}\left(h_{1}^{\uparrow+}\right)=\operatorname{Card}\left(h_{1}^{\uparrow+}\right) \geq \delta_{\varepsilon}(m-1)$. Since $g$ and $h$ are opposite with respect to $f$, we derive from Lemma 7 that $\operatorname{Card}\left(f_{1}^{\uparrow+}\right)=\operatorname{Card}\left(g_{1}^{\uparrow+}\right)+\operatorname{Card}\left(h_{1}^{\uparrow+}\right)$. Hence, $\operatorname{Card}^{+}\left(f_{1}^{\uparrow+}\right) \geq$
$\operatorname{Card}\left(f_{1}^{\uparrow+}\right) \geq 2 \delta_{\varepsilon}(m-1)=\delta_{\varepsilon}(m)$. Then (ii) holds.

Second case: $\varepsilon(\operatorname{dim}(f)+1)=1$. If $\operatorname{Card}^{-}\left(f_{1}^{\uparrow+}\right) \geq \delta_{\varepsilon}(m)=$ $2 \delta_{\varepsilon}(m-1)-1$, then (from Lemma 7) in each couple of opposite faces with respect to $f$ there exists a face $g$ such that $\operatorname{Card}^{-}\left(g^{\uparrow+}\right)=\operatorname{Card}\left(g_{1}^{\uparrow+}\right) \geq \delta_{\varepsilon}(m-1)$, that is, thanks to the induction hypothesis, in each couple of opposite faces with respect to $f$ there exists a face whose value is equal to 1 . Thus, $\mu(f)=\bigwedge_{\{a, b\} \in \mathrm{opp}(f)} \mu(a) \vee \mu(b) \geq \bigwedge_{\{a, b\} \in \mathrm{opp}(f)} 1=1$. Then $(i)$ holds. Conversely, if $\mu(f)=1$, thanks to Lemma 1 and the induction hypothesis, we know that in each couple of opposite faces with respect to $f$ there exists a face $g$ such that $\operatorname{Card}^{+}\left(g^{\uparrow+}\right)=\operatorname{Card}\left(g_{1}^{\uparrow+}\right) \geq \delta_{\varepsilon}(m-1)$. We deduce from Lemma 9 that $\operatorname{Card}\left(f_{1}^{\uparrow+}\right) \geq 2 \delta_{\varepsilon}(m-1)-1=\delta_{\varepsilon}(m)$. Then $(i i)$ holds.

From Proposition 10 and Definition 1 (needed when $f_{1}^{\uparrow+}$ is a trihedron), we derive the following corollary.

Corollary 1 Let $\mu: \mathbb{F}^{n} \rightarrow\{0,1\}$ be an $\varepsilon$-regular image. Let $f$ be a $k$-face of $\mathbb{F}^{n}$ (with $\left.n-3 \leq k \leq n-1\right)$. Then $\mu(f)=1$ iff the set $f_{1}^{\uparrow+}$ satisfies the condition $C_{\varepsilon}$ given in Table 2 .

\subsection{Regular images and regular open/closed sets}

We have defined the object (resp. the background) of a regular image $\mu: \mathbb{F}^{n} \rightarrow\{0,1\}$ as the set $\mu^{-1}(\{1\})\left(\right.$ resp. $\left.\mu^{-1}(\{0\})\right)$. 
We show in this section that they are topologically regular, i.e., they do not have thin parts nor thin holes (by "thin", we mean of lower dimension than the surrounding space).

Lemma 2 Let $\mu: \mathbb{F}^{n} \rightarrow\{0,1\}$ be an $\varepsilon$-regular image. Let $x \in\{0,1\}$ Then, we have

$$
\left(\left(\mu^{-1}(\{x\})\right)^{\downarrow}\right)^{\circ} \subseteq \mu^{-1}(\{x\}) \subseteq\left(\left(\mu^{-1}(\{x\})\right)^{\circ}\right)^{\downarrow}
$$

Proof The proof is proposed for $x=1$. The case $x=0$ is obtained by duality (see Remark 1). Let $E$ be a subset of $\mathbb{F}^{n}$. The two following chains of implications are easy to establish:

$$
\begin{aligned}
& f \in\left(E^{\downarrow}\right)^{\circ} \Rightarrow f^{\uparrow} \subseteq E^{\downarrow} \Rightarrow f^{\uparrow+} \subseteq E^{\downarrow} \Rightarrow f^{\uparrow+} \subseteq E \\
& \exists g \in f^{\uparrow+}, g \in E \Rightarrow \exists g \geqslant f, g \in E^{\circ} \Rightarrow f \in\left(E^{\circ}\right)^{\downarrow}
\end{aligned}
$$

Now, we take $E=\mu^{-1}(\{1\})$. From Proposition 6, we have $f^{\uparrow+} \subseteq E \Rightarrow f \in E$. From Proposition 5, we also have $f \in$ $E \Rightarrow \exists g \in f^{\uparrow+}, g \in E$. Hence, the assertion holds: $E^{\downarrow^{\triangleright} \subseteq}$ $E \subseteq E^{\circ \downarrow}$.

Proposition 11 Let $\mu: \mathbb{F}^{n} \rightarrow\{0,1\}$ be an $\varepsilon$-regular image. Let $x \in\{0,1\}$. Then $\left(\mu^{-1}(\{x\})\right)^{\circ}$ is a regular open set and $\left(\mu^{-1}(\{x\})\right)^{\downarrow}$ is a regular closed set.

Proof The proof is proposed for $x=1$. The case $x=0$ is obtained by duality (see Remark 1 ). Let $E=\mu^{-1}(\{1\})$. From Lemma 2, we have $\left(E^{\downarrow}\right)^{\circ} \subseteq E \subseteq\left(E^{\circ}\right)^{\downarrow}$. Hence, we readily derive:

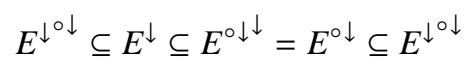

where the last inclusion comes from $E \subseteq E^{\downarrow}$. Therefore, we get the equality $E^{\downarrow^{\triangleright \downarrow}}=E^{\downarrow}$ which means that $E^{\downarrow}$ is a regular closed set. The proof of the equality $E^{\circ \downarrow^{\circ}}=E^{\circ}$ is similar.

Corollary 2 Let $\mu: \mathbb{F}^{n} \rightarrow\{0,1\}$ be an $\varepsilon$-regular image. If $\varepsilon=-1$, then $\mu^{-1}(\{1\})$ (resp. $\left.\mu^{-1}(\{0\})\right)$ is a regular open (resp. closed) set. If $\varepsilon=1$, then $\mu^{-1}(\{1\})\left(\right.$ resp. $\left.\mu^{-1}(\{0\})\right)$ is a regular closed (resp. open) set.

Proof The proof is proposed for $x=1$. The case $x=0$ is obtained by duality (see Remark 1). If $\varepsilon=-1$, we readily have $g \geqslant f \Rightarrow g^{\uparrow+} \subseteq f^{\uparrow+} \Rightarrow \bigwedge_{h \in g^{\uparrow+}} \mu(h) \geq \bigwedge_{h \in f^{\uparrow+}} \mu(h) \Rightarrow \mu(g) \geq$ $\mu(f)$. Thus, $g \geqslant f$ and $f \in \mu^{-1}(\{1\})$ imply $g \in \mu^{-1}(\{1\})$, that is $\mu^{-1}(\{1\})$ is an open set. It follows that $\mu^{-1}(\{1\})=$ $\left(\mu^{-1}(\{1\})\right)^{\circ}$ is a regular open set. Similarly if $\varepsilon=1, g \leqslant f$ implies $\mu(g) \geq \mu(f)$. Hence, $\mu^{-1}(\{1\})$ is a regular closed set.

\section{Paths and (digital) fundamental groups}

In this section, we study how the functions $\zeta_{\varepsilon}$ defined in Section 3 behave relatively to the classical notions of path in $\mathbb{Z}^{n}$ and $\mathbb{F}^{n}$. The main results are Theorems 3 and 4 . Theorem 3 states that $\zeta_{\varepsilon}$ induces a bijection between the set of the connected components of the object (resp. background) $\lambda^{-1}(\{1\}) \subseteq \mathbb{Z}^{n}$ (resp. $\lambda^{-1}(\{0\}) \subseteq \mathbb{Z}^{n}$ ) associated to an image $\lambda: \mathbb{Z}^{n} \rightarrow\{0,1\}$, and the set of the connected components of the object (resp. background) $\mu^{-1}(\{1\}) \subseteq \mathbb{F}^{n}$ (resp. $\mu^{-1}(\{0\}) \subseteq$ $\left.\mathbb{F}^{n}\right)$ associated to the regular image $\mu: \mathbb{F}^{n} \rightarrow\{0,1\}$, defined by $\mu=\zeta_{\varepsilon}(\lambda)$, the function $\varepsilon$ being chosen with respect to a given pair of adjacencies in $\mathbb{Z}^{n}$. Theorem 4 states that $\zeta_{\varepsilon}$ induces an isomorphism between the digital fundamental group of $\lambda^{-1}(\{1\})\left(\right.$ resp. $\left.\lambda^{-1}(\{0\})\right)$ and the fundamental group of $\mu^{-1}(\{1\})\left(\right.$ resp. $\left.\mu^{-1}(\{0\})\right)$.

\subsection{Background notions on paths and arcs}

We recall the classical definitions of path equivalence, fundamental group in a topological space (in particular, in a poset), and of digital path equivalence and digital fundamental group in $\mathbb{Z}^{n}$.

\subsubsection{The fundamental group of topological spaces}

Let $X$ be a topological space. A path $p$ in $X$ is a continuous function $p:[0,1] \rightarrow X$. Two paths $p, q$ in $X$ are equivalent if they have the same extremities (i.e., $p(0)=q(0)$ and $p(1)=$ $q(1))$ and $p$ can be continuously deformed to fit $q$, that is if there exists a continuous map $h:[0,1] \times[0,1] \rightarrow X$ such that, for all $t \in[0,1], h(t, 0)=p(t)$ and $h(t, 1)=q(t)$, and, for all $u \in[0,1], h(0, u)=p(0)=q(0)$ and $h(1, u)=p(1)=$ $q(1)$ (the map $h$ is called a path-homotopy). This relation on paths is actually an equivalence relation. We write $[p]$ for the equivalence class of $p$. If $p, q$ are two paths in $X$ such that $p(1)=q(0)$ we can define the product $p \cdot q$ by

$(p \cdot q)(t)= \begin{cases}p(2 t) & \text { if } t \in\left[0, \frac{1}{2}\right] \\ q(2 t-1) & \text { if } t \in\left[\frac{1}{2}, 1\right]\end{cases}$

This product is well defined on equivalence classes by $[p]$. $[q]=[p \cdot q]$. Let $x$ be a point of $X$. A loop at $x$ is a path in $X$ which starts and ends at $x$. The product of two loops at $x$ is a loop at $x$ and the set $\pi(X, x)$ of equivalence classes of loops at $x$ is a group for this product. It is called the fundamental group of $X$ (with basepoint $x$ ).

\subsubsection{Finite paths in posets}

In a poset $X$, a function $f:[0,1] \rightarrow X$ is a step function if there exist finitely many intervals $\left(I_{i}\right)_{i=0}^{r}(r \geq 0)$ such that $f$ is constant on each interval $I_{i}$ and $[0,1]=\bigcup_{i=0}^{r} I_{i}$. If for all $i \in \llbracket 1, r \rrbracket, \sup \left(I_{i-1}\right)=\inf \left(I_{i}\right)$ and $f\left(I_{i-1}\right) \neq f\left(I_{i}\right)$, we write $f=\sum_{i=0}^{r} x_{i} \mathbf{1}_{I_{i}}$ where $\left\{x_{i}\right\}=f\left(I_{i}\right)$. A finite path in $X$ is a path in $X$ which is a step function. The sequence $\left(I_{i}\right)_{i=0}^{r}$ is called the intervals sequence of $p$ and the sequence $\left(x_{i}\right)_{i=0}^{r}$ the track of $p$. A finite path is regular if there is no singleton in its intervals sequence. Two comparable paths (for the componentwise order), finite or not, with same extremities 
are equivalent. In the sequel, we denote by $\leqslant$ the componentwise order on paths. The next four results have been proved in [37].

Proposition 12 The track of a finite path is an arc and any arc is the track of a regular finite path.

Proposition 13 A step function $p=\sum_{i=0}^{r} x_{i} \mathbf{1}_{I_{i}}$ is a finite path in $X$ iff for all $i \in \llbracket 0, r-1 \rrbracket, x_{i}$ is adjacent to $x_{i+1}$ and $x_{i} \leqslant x_{i+1} \Leftrightarrow \sup \left(I_{i}\right) \in I_{i}$.

The product of two arcs $\left(x_{i}\right)_{i=0}^{r}$ and $\left(y_{i}\right)_{i=0}^{s}$ is defined by $\left(x_{i}\right)_{i=0}^{r} \cdot\left(y_{i}\right)_{i=0}^{s}=\left(x_{0}, \ldots, x_{r}, y_{1}, \ldots, y_{s}\right)$ provided that $x_{r}=y_{0}$. An $\operatorname{arc} \chi=\left(x_{i}\right)_{i=0}^{r}(r \geq 2)$ is an elementary stretching (in $X)$ of an arc $\chi^{\prime}$ if for some $j \in \llbracket 1, r-1 \rrbracket, \chi^{\prime}=\left(x_{i}\right)_{i=0, i \neq j}^{r}$ or $\left(x_{j-1}=x_{j+1}\right.$ and $\left.\chi^{\prime}=\left(x_{i}\right)_{i=0, i \neq j-1, i \neq j}^{r}\right)$. An arc $\chi$ is a $d e-$ formation of an arc $\chi^{\prime}$ if there exists a sequence $\left(\chi_{i}\right)_{i=0}^{s}$ of arcs in $X$ such that $\chi_{0}=\chi, \chi_{s}=\chi^{\prime}$ and for any $i \in \llbracket 1, s \rrbracket$, either $\chi_{i}$ is an elementary stretching of $\chi_{i-1}$ or vice versa. Let $x$ be a point in $X$. "Being a deformation or equal" is an equivalence relation in the set of $\operatorname{arcs}$ in $X$ from $x$ to $x$. The set of equivalence classes, denoted by $\rho(X, x)$, is a group for the arc product.

Theorem 1 Two finite paths are equivalent iff they have the same track or the track of one of them is a deformation of the track of the other.

Theorem 2 Let $x \in X$. The fundamental group $\pi(X, x)$ of $X$ with basepoint $x$ is isomorphic to the group $\rho(X, x)$.

\subsubsection{The digital fundamental group of $\mathbb{Z}^{n}$}

A discrete analogue of the concept of fundamental group has been proposed in digital topology [4]. Let $n \in\{2,3\}$ and $X \subseteq \mathbb{Z}^{n}$. The definition of the product for digital paths is straightforward but not so is the notion of equivalence between digital paths or loops. Two paths $\left(a_{i}\right)_{i=0}^{r},\left(b_{i}\right)_{i=0}^{s}(r, s \geq$ 0 ) with same extremities are directly equivalent (in $X)^{7}$ if they differ only in a unit lattice cube of $\mathbb{Z}^{n}$ provided that, if $n=3$ and the pair of adjacencies is $(6,26)$, the cube must not contain two diametrically opposite points not in $X$.

Finally, two paths $p_{0}, p_{t}(t \geq 0)$ with same extremities are equivalent (in $X$ ) if there is a sequence $\left(p_{i}\right)_{i=0}^{t}$ of paths such that, for all $i \in \llbracket 1, t \rrbracket, p_{i}$ is directly equivalent to $p_{i-1}$.

${ }^{7}$ In [11], we find another (equivalent) form of the previous definition. Two paths $p, q$ are directly equivalent if there exist four paths $p_{0}, p_{1}, p_{1}^{\prime}, p_{2}$ such that $p=p_{0} \cdot p_{1} \cdot p_{2}, q=p_{0} \cdot p_{1}^{\prime} \cdot p_{2}, p_{1}$ and $p_{1}^{\prime}$ have the same extremities and are both included in a $2 \times 2$ square if $n=2$ or $n=3$ and the pair of adjacencies is $(6,26)$, or in a $2 \times 2 \times 2$ cube otherwise.
4.2 Mapping paths in $\mathbb{Z}^{n}$ onto arcs in $\mathbb{F}^{n}$

Let $\chi$ and $\chi^{\prime}$ be two arcs in $\mathbb{F}^{n}$. We write ${ }^{8} \chi \leqslant \chi^{\prime}$ if there exist two paths $p \leqslant p^{\prime}$ in $\mathbb{F}^{n}$ whose tracks are $\chi$ and $\chi^{\prime}$ (all paths in $\mathbb{F}^{n}$ considered in the sequel are regular finite paths).

Definition 2 Let $\omega$ be an adjacency relation on $\mathbb{Z}^{n}$ and $\gamma=$ $\left(p_{i}\right)_{i=0}^{r}(r \geq 0)$ be an $\omega$-path in $\mathbb{Z}^{n}$. We define the arc $\zeta(\gamma)$ in $\mathbb{F}^{n}$ by $\zeta(\gamma)=\left(q_{j}\right)_{j=0}^{2 r}$ with $q_{j}=\dot{p}_{\frac{j}{2}}$ if $j$ is even and $q_{j}=$ $q_{j-1} \cap q_{j+1}$ if $j$ is odd, for all $j \in \llbracket 0,2 r \rrbracket$.

It is obvious that the sequence of faces $\zeta(\gamma)$ defined above is actually an arc in $\mathbb{F}^{n}$ which is itself the track of a regular finite path in $\mathbb{F}^{n}$ (Proposition 12).

The following proposition states that $\zeta$ associates to a path in the object (resp. in the background), of a digital image $\lambda$, an arc in the object (resp. in the background) of the complex image $\zeta_{\varepsilon}(\lambda)$ under the condition that the connectivity function $\varepsilon$ has been well chosen. The main consequence of this proposition is that the images of the connected components of the digital object (resp. background) are included in the connected components of the image of the object (resp. background).

Proposition 14 Let $(\alpha, \beta)$ be a pair of adjacencies on $\mathbb{Z}^{n}$. Let $\varepsilon$ be the connectivity function associated to $(\alpha, \beta)$ (see Table 1). Let $x \in\{0,1\}$. Let $\omega=\alpha$ if $x=1$ and $\omega=\beta$ if $x=0$. Let $\lambda: \mathbb{Z}^{n} \rightarrow\{0,1\}$ be an image in $\mathbb{Z}^{n}$ and $\mu=\zeta_{\varepsilon}(\lambda)$ be the corresponding image in $\mathbb{F}^{n}$. Let $\gamma$ be an $\omega$-path in $\lambda^{-1}(\{x\})$. Then, $\zeta(\gamma)$ is an arc in $\mu^{-1}(\{x\})$.

Proof The proof is proposed for $(\alpha, \beta)=\left(3^{n}-1,2 n\right)$ and $(n=3$ and $(\alpha, \beta)=(18,6))$. The cases $(\alpha, \beta)=\left(2 n, 3^{n}-1\right)$ and $(n=3$ and $(\alpha, \beta)=(6,18))$ are obtained by duality (see Remark 1).

Case $(\alpha, \beta)=\left(3^{n}-1,2 n\right)$. Here, $\varepsilon(k)=1$ for all $k \in \llbracket 1, n \rrbracket$. If $\gamma=\left(p_{i}\right)_{i=0}^{r}(r \geq 0)$ is an $\alpha$-path in $\lambda^{-1}(\{1\})$, from Proposition 8 , we derive directly that $\mu\left(p_{i-1}^{\dot{ }} \cap \dot{p}_{i}\right)=1$ for all $i \in \llbracket 1, r \rrbracket$, that is $\zeta(\gamma)$ is an $\operatorname{arc}$ in $\mu^{-1}(\{1\})$. If $\gamma$ is a $\beta$-path in $\lambda^{-1}(\{0\})$, then $\operatorname{dim}\left(p_{i-1} \cap \dot{p}_{i}\right)=n-1$ and thus $\mu\left(p_{i-1} \cap \dot{p}_{i}\right)=$ $\mu\left(p_{i-1}\right) \vee \mu\left(\dot{p}_{i}\right)=0 \vee 0=0$ for all $i \in \llbracket 1, r \rrbracket$. So, $\zeta(\gamma)$ is an $\operatorname{arc~in~} \mu^{-1}(\{0\})$.

Case $n=3$ and $(\alpha, \beta)=(18,6)$. The connectivity function $\varepsilon$ is such that $\varepsilon(3)=1$ and $\varepsilon(2)=-1$. If $\gamma$ is an 18-path in $\lambda^{-1}(\{1\})$, then all the faces of $\gamma$ have a dimension greater or equal to 1 , thereby we can use the direct part of Proposition 8 to conclude that $\zeta(\gamma)$ is an arc in $\mu^{-1}(\{1\})$. If $\gamma$ is a 6-path in $\lambda^{-1}(\{0\})$, we use the proof of the case $(\alpha, \beta)=\left(3^{n}-1,2 n\right)$.

The following proposition is straightforward.

8 The notation $\chi \leqslant \chi^{\prime}$ does not mean that $\leqslant$ is an order (it is actually only a pre-order on regular finite paths). For instance, if $a \leqslant b \leqslant c$ are three distinct faces of $\mathbb{F}^{n}$, it can readily be proved that $(a, c) \leqslant$ $(a, b, c) \leqslant(a, c)$.

${ }^{9}$ In this article, the terminology "complex image" denotes a subset of $\mathbb{F}^{n}$. 
Proposition 15 Let $\omega$ be an adjacency relation on $\mathbb{Z}^{n}$. The corresponding function $\zeta$ is a homomorphism for the paths product and the arc product: for all $\omega$-paths $\gamma, \gamma^{\prime} \in \mathbb{Z}^{n}$, $\zeta\left(\gamma \cdot \gamma^{\prime}\right)=\zeta(\gamma) \cdot \zeta\left(\gamma^{\prime}\right)$.

The injectivity of $\zeta$ is obvious since two distinct $n$-xels $a, b \in \mathbb{Z}^{n}$ are associated to distinct facets $\dot{a}, \dot{b} \in \mathbb{F}^{n}$. Proposition 16 establishes the surjectivity up to deformations: any $\operatorname{arc} \chi$ from $\dot{a}$ to $\dot{b}$ in an object (resp. in the background) of the complex image is the deformation of an $\operatorname{arc} \zeta(\gamma)$ for some path $\gamma$ from $a$ to $b$ of the object (resp. background) of its associated digital image (if the complex image is associated to such a digital image). Lemma 3 is the basic block for the proof of Proposition 16.

Lemma 3 Let $(\alpha, \beta)$ be a pair of adjacencies on $\mathbb{Z}^{n}$. Let $\varepsilon$ be the connectivity function associated to $(\alpha, \beta)$. Let $x \in\{0,1\}$. Let $\omega=\alpha$ if $x=1$ and $\omega=\beta$ if $x=0$. Let $\lambda: \mathbb{Z}^{n} \rightarrow\{0,1\}$ be an image in $\mathbb{Z}^{n}$ and $\mu=\zeta_{\varepsilon}(\lambda)$ be the corresponding image in $\mathbb{F}^{n}$. Let $p$ be a path in $\mu^{-1}(\{x\})$ whose track is $(f, g, h)$ with $f \supset g \subset h$. Let $a, b \in \mathbb{Z}^{n}$ be two xels in $\lambda^{-1}(\{x\})$ such that $\dot{a} \supset f$ and $h \subset \dot{b}$. Then, there exists an $\omega$-path $\gamma$ in $\lambda^{-1}(\{x\})$ from a to $b$ and a path $q, q \geqslant p$, whose track is $\zeta(\gamma)$.

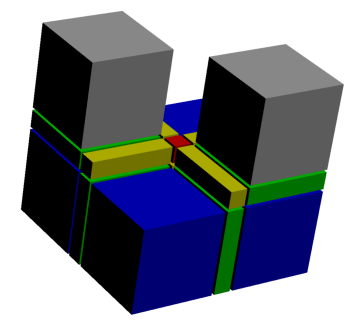

Fig. 12 In grey and red: an arc in $\mathbb{F}^{3}$ in a $(-1,1,-1)$-regular image. In this arc, two consecutive grey facets share a 0 -face (in red) but their intersection does not belong to the object.

Proof The proof is proposed for $x=1$. The case $x=0$ is obtained by duality (see Remark 1). If $a=b$, we set $\gamma=(a)$ and $q=\dot{a} \mathbf{1}_{[0,1]}$ and we are done. Let us now suppose that $a \neq b$. We set $g^{\prime}=\dot{a} \cap \dot{b}$. Let $p=f \mathbf{1}_{I}+g \mathbf{1}_{J}+h \mathbf{1}_{K}$ (where $I, J, K$ are intervals included in $[0,1])$ be a path. Since $\dot{a} \supset$ $f \supset g \subset h \subset \dot{b}$, we have $g \subset g^{\prime}$. If $\varepsilon=-1$, since $g \in$ $\mu^{-1}(\{1\})$, from Proposition 1 we derive that all facets in $g^{\uparrow}$ have value 1 and, therefore, all facets in $g^{\prime \uparrow}$ have value 1 . Thus, $g^{\prime}$ is in $\mu^{-1}(\{1\})$. If $\varepsilon=1$, still from Proposition 1 , we derive that $g^{\prime} \in \mu^{-1}(\{1\})$ since there exists at least one facet in $\mu^{-1}(\{1\}) \cap g^{\prime \uparrow}$. If $n=3$ and $\varepsilon(3)=1$, we derive from Proposition 8 that if $\operatorname{dim}\left(g^{\prime}\right) \geq 1$, then $g^{\prime} \in \mu^{-1}(\{1\})$ and if $\operatorname{dim}\left(g^{\prime}\right)=0$, then $g^{\prime}=g$ so, $g^{\prime} \in \mu^{-1}(\{1\})$. In all the previous cases, we set $\gamma_{0}=(a, b)$ and $q_{0}=\dot{a} \mathbf{1}_{I}+g^{\prime} \mathbf{1}_{J}+\dot{b} \mathbf{1}_{K}$. Observe, before examining the last case, that we cannot claim that $\gamma_{0}$ is an $\omega$-path, but just a $\left(3^{n}-1\right)$-path, since we do not know if $a$ and $b$ are $\omega$-adjacent. Now, let us consider the case $n=3$ and $\varepsilon=(-1,1,-1)$ which corresponds to $(\alpha, \beta)=$ $(6,18)$. In this case, if $\operatorname{dim}\left(g^{\prime}\right)=1$ and $\operatorname{dim}(g)=0$, it may happen that $g^{\prime} \notin \mu^{-1}(\{1\})$ (see Figure 12). Let us suppose that $g^{\prime} \notin \mu^{-1}(\{1\})$ (and thus, $\operatorname{dim}\left(g^{\prime}\right)=1$ and $\operatorname{dim}(g)=0$ ). From Proposition 10 and Table 2, we know that there are at least 6 facets in $g_{1}^{\uparrow+}$ and at most 2 facets in $g_{1}^{\prime \uparrow+}$ (and actually, there are two facets in $g_{1}^{\prime \uparrow}, \dot{a}$ and $\dot{b}$ ). It follows that the facet $\dot{c}$, whose intersection with $\dot{a}$ is $g$, is in $\mu^{-1}(\{1\})$ (see Figure 12 to look at the only possible configuration), and $\dot{c}$ shares a 2-face with $\dot{b}$. We call this 2-face $h^{\prime}$. It is plain that $h^{\prime} \in$ $\mu^{-1}(\{1\})$. So, we set $\gamma_{0}=(a, c, b)$ and $q_{0}=\dot{a} \mathbf{1}_{I}+g \mathbf{1}_{J_{1}}+\dot{c} \mathbf{1}_{J_{2}}+$ $h^{\prime} \mathbf{1}_{J_{3}}+\dot{b} \mathbf{1}_{K}$ where $J_{1} \cup J_{2} \cup J_{3}=J, \max \left(J_{1}\right)=\inf \left(J_{2}\right)$, and $\sup \left(J_{2}\right)=\min \left(J_{3}\right)$. As previously observed, it may happen that, for $y=g^{\prime}$ or $y=g, \operatorname{dim}(y) \leq n-k$ where $k=2$ if $\alpha=2 n, k=3$ if $\alpha=18$. Let us write $\dot{d}$ for the facets following $y$ in $\zeta\left(\gamma_{0}\right)(d=b$ or $d=c)$. From Proposition 9, we derive that there exists an arc $\chi=\zeta\left(\gamma_{1}\right)$ from $\dot{a}$ to $\dot{d}$ in $y^{\uparrow}$ where $\gamma_{1}$ is an $\omega$-path from $a$ to $d$. By introducing, if necessary, this path $\gamma_{1}$ between $a$ and $b$ if $\gamma_{0}=(a, b)$, or between $a$ and $c$ if $\gamma_{0}=(a, c, b)$, we build in $\lambda^{-1}(\{1\})$ an $\omega$-path $\gamma$ from $a$ to $b$. Since this $\chi$ is in $y^{\uparrow}$, one can readily build in $\mu^{-1}(\{1\})$ a path $q \geqslant q_{0}$ whose track is $\zeta(\gamma)$. Hence, the result holds.

Proposition 16 Let $(\alpha, \beta)$ be a pair of adjacencies on $\mathbb{Z}^{n}$. Let $\varepsilon$ be the connectivity function associated to $(\alpha, \beta)$. Let $x \in\{0,1\}$. Let $\omega=\alpha$ if $x=1$ and $\omega=\beta$ if $x=0$. Let $\lambda: \mathbb{Z}^{n} \rightarrow\{0,1\}$ be an image in $\mathbb{Z}^{n}$ and $\mu=\zeta_{\varepsilon}(\lambda)$ be the corresponding image in $\mathbb{F}^{n}$. Let $a, b \in \mathbb{Z}^{n}$. Let $\chi$ be an arc from the facet $\dot{a}$ to the facet $\dot{b}$ in $\mu^{-1}(\{x\})$. Then, there exists an $\omega$-path $\gamma$ from a to $b$ in $\lambda^{-1}(\{x\})$ such that $\zeta(\gamma)$ is a deformation in $\mu^{-1}(\{x\})$ of $\chi$ in $\mu^{-1}(\{x\})$. Moreover, if $p$ is a path in $\mu^{-1}(\{x\})$ whose track is $\chi$, there exists a path $q$ in $\mu^{-1}(\{x\})$, $q \geqslant p$, whose track is $\zeta(\gamma)$.

Proof The proof is proposed for $x=1$. The case $x=0$ is obtained by duality (see Remark 1). Let $\chi=\left(q_{i}\right)_{i=0}^{r}(r \geq 0)$ be an arc from the facet $\dot{a}$ to the facet $\dot{b}$ in $\mu^{-1}(\{1\})$ and $p=\sum_{i=0}^{r} q_{i} \mathbf{1}_{I_{i}}$ be a path whose track is $\chi$ (Proposition 12). We build the proof by induction on $r$, the size of $\chi$. If $r=0$, the statement is obvious. Let us now suppose that the property is true whenever the size of $\chi$ is strictly less than an integer $r \geq 2$ ( $r=1$ is impossible). Let $j \in \llbracket 1, r-1 \rrbracket$ be the lowest integer such that $q_{j}$ is a local minimum of $\chi$, i.e., such that $q_{j-1} \supset q_{j} \subset q_{j+1}$ (remember that $q_{0}=\dot{a}$ and $q_{r}=\dot{b}$ are facets) and let $k \in \llbracket j+1, r \rrbracket$ be the lowest integer such that $q_{k}$ is a local maximum of $\chi$, i.e. $q_{k-1} \subset q_{k} \supset q_{k+1}$. Let $t$ be the centre of the interval $I_{k}$ and $\varphi:[0,1] \rightarrow[0,1]$ be the bijective function which puts $t$ on $\frac{1}{2}$ and is linear between 0 and $t$ and between $t$ and 1. We have $p \circ \varphi^{-1}=$ $\left(\sum_{i=0}^{k} q_{i} \mathbf{1}_{(2 \varphi)\left(I_{i}^{\prime}\right)}\right) .\left(\sum_{i=k}^{r} q_{i} \mathbf{1}_{(2 \varphi-1)\left(I_{i}^{\prime \prime}\right)}\right)$ where $I_{i}^{\prime}=I_{i}$ if $0 \leq i<k$, 
$I_{k}^{\prime}=I_{k} \cap[0, t], I_{k}^{\prime \prime}=I_{k} \cap[t, 1]$ and $I_{i}^{\prime \prime}=I_{i}$ if $k<i \leq r$. From Proposition 5, we derive that there exists a xel $c \in \mathbb{Z}^{n}$, such that $c \in \lambda^{-1}(\{1\})$ and $q_{k} \subseteq \dot{c}$ (if $q_{k}$ is a facet of $\mathbb{F}^{n}, \dot{c}=q_{k}$ ). As $\dot{c} \mathbf{1}_{(2 \varphi-1)\left(I_{k}^{\prime \prime}\right)}+\sum_{i=k+1}^{r} q_{i} \mathbf{1}_{(2 \varphi-1)\left(I_{i}^{\prime \prime}\right)}$ is a path from $\dot{c}$ to $\dot{b}$ whose length is strictly lower than $r$, by the induction hypothesis, there exists an $\omega$-path $\gamma^{(2)}$ from $c$ to $b$ in $\lambda^{-1}(\{1\})$ and a path $q^{(2)}$ from $\dot{c}$ to $\dot{b}$ such that $\zeta\left(\gamma^{(2)}\right)$ is the track of $q^{(2)}$ and $q^{(2)} \geqslant \dot{c} \mathbf{1}_{(2 \varphi-1)\left(I_{k}^{\prime \prime}\right)}+\sum_{i=k+1}^{r} q_{i} \mathbf{1}_{(2 \varphi-1)\left(I_{i}^{\prime \prime}\right)} \geqslant \sum_{i=k}^{r} q_{i} \mathbf{1}_{(2 \varphi-1)\left(I_{i}^{\prime \prime}\right)}$. On the other side, from Lemma 3, we can define in $\lambda^{-1}(\{1\})$ an $\omega$-path $\gamma^{(1)}$ from $a$ to $c$ and a path in $\mu^{-1}(\{1\}), q^{(1)} \geqslant$ $\dot{a} \mathbf{1}_{(2 \varphi)(I)}+q_{j} \mathbf{1}_{(2 \varphi)\left(I_{j}^{\prime}\right)}+\dot{c} \mathbf{1}_{(2 \varphi)(J)} \geqslant \sum_{i=0}^{k} q_{i} \mathbf{1}_{(2 \varphi)\left(I_{i}^{\prime}\right)}$, where $I=$ $\bigcup_{i=0}^{j-1} I_{i}^{\prime}$ and $J=\bigcup_{i=j+1}^{k} I_{i}^{\prime}$, whose track is $\zeta\left(\gamma^{(1)}\right)$. Eventually, we set $\gamma=\gamma^{(1)} \cdot \gamma^{(2)}$ and $q=q^{(1)} \cdot q^{(2)}$. As $\zeta$ is a morphism (Proposition 15), $\zeta(\gamma)$ is the track of $q$ and from Lemma 10, we have $q \geqslant p \circ \varphi^{-1}$, thereby $q \circ \varphi \geqslant p$. Of course, $q \circ \varphi$ has the same track as $q$, so the result holds: since $q \circ \varphi \geqslant p$, the track $\zeta(\gamma)$ of $q \circ \varphi$ is a deformation of the track $\chi$ of $p$ (Theorem 1).

Theorem 3 Let $(\alpha, \beta)$ be a pair of adjacencies on $\mathbb{Z}^{n}$. Let $\varepsilon$ be the connectivity function associated to $(\alpha, \beta)$. Let $x \in$ $\{0,1\}$. Let $\lambda: \mathbb{Z}^{n} \rightarrow\{0,1\}$ be an image in $\mathbb{Z}^{n}$ and $\mu=\zeta_{\varepsilon}(\lambda)$ be the corresponding image in $\mathbb{F}^{n}$. Then the function which associates to the xel $a \in \mathbb{Z}^{n}$ the facet $\dot{a} \in \mathbb{F}^{n}$ induces a oneto-one correspondence between the connected components of $\lambda^{-1}(\{x\})$ and the connected components of $\mu^{-1}(\{x\})$

Proof The proof is proposed for $x=1$. The case $x=0$ is obtained by duality (see Remark 1$)$. Let $\equiv_{\lambda}\left(\right.$ resp. $\left.\equiv_{\mu}\right)$, be the equivalence relation defined on $\lambda^{-1}(\{1\})$ (resp. $\mu^{-1}(\{1\})$ ), by $a \equiv_{\lambda} b$ (resp. $a \equiv_{\mu} b$ ), iff $a$ and $b$ belong to the same connected component of $\lambda^{-1}(\{1\})$ (resp. $\left.\mu^{-1}(\{1\})\right)$, i.e., there exists a path from $a$ to $b$ in $\lambda^{-1}(\{1\})$ (resp. $\mu^{-1}(\{1\})$ ). From Proposition 14, we can define the quotient map $\dot{\zeta}$ from the set of connected components of $\lambda^{-1}(\{1\})$ to the set of connected components of $\mu^{-1}(\{1\})$. Let $C$ be a connected component of $\mu^{-1}(\{1\})$ and $f$ be a face in $C$. From Proposition 5, we derive that $f$ is at least included in one facet $\dot{a} \in \mu^{-1}(\{1\})$ so $\dot{a}=\zeta(a) \in C$ (for $(f, a)$ is an arc). Hence, $C=\dot{\zeta}\left(C_{a}\right)$ where $C_{a}$ is the connected component of $\lambda^{-1}(\{1\})$ including $a$ and, as we have not made any hypothesis on $C, \dot{\zeta}$ is surjective. Let $a, b \in \mathbb{Z}^{n}$ be to xels such that $\dot{a} \equiv_{\mu} \dot{b}$. Proposition 16 indicates that there exists an $\omega$-path from $a$ to $b$ in $\lambda^{-1}(\{1\})$ so $a \equiv_{\lambda} b$. This establishes the injectivity of $\dot{\zeta}$, so $\dot{\zeta}$ is a bijection.

\subsection{Fundamental groups}

The aim of this section is to compare the digital fundamental group defined by Kong [4] for subsets of $\mathbb{Z}^{n}, n \in\{2,3\}$, with the fundamental group of subspaces of $\mathbb{F}^{n}$. Thanks to Theorem 2, we can use arcs as well as paths in $\mathbb{F}^{n}$ in order to perform this comparison.
Proposition 17 Let $(\alpha, \beta)$ be a pair of adjacencies on $\mathbb{Z}^{n}$. Let $\varepsilon$ be the connectivity function associated to $(\alpha, \beta)$. Let $x \in\{0,1\}$. Let $\omega=\alpha$ if $x=1$ and $\omega=\beta$ if $x=0$. Let $\lambda: \mathbb{Z}^{n} \rightarrow\{0,1\}$ be an image in $\mathbb{Z}^{n}$ and $\mu=\zeta_{\varepsilon}(\lambda)$ be the corresponding image in $\mathbb{F}^{n}$. Let $\gamma, \gamma^{\prime}$ be two equivalent $\omega$ paths in $\lambda^{-1}(\{x\})$. Then, $\zeta\left(\gamma^{\prime}\right)$ is a deformation of $\zeta(\gamma)$ in $\mu^{-1}(\{x\})$.

Proof The proof is proposed for $x=1$. The case $x=0$ is obtained by duality (see Remark 1). Since $\zeta$ is a homomorphism (Proposition 15) and "being a deformation" is an equivalence relation, we derive from the definition of digital homotopy equivalence (see section 4.1.3) that it suffices to establish the lemma for two digital paths $\gamma, \gamma^{\prime}$ having the sames extremities and included in a $2 \times 2$ square or in a $2 \times 2 \times 2$ cube. That is, the loop $\zeta\left(\gamma \cdot \gamma^{\prime-1}\right)$ can be deformed (in $\mu^{-1}(\{x\})$ ) in a constant arc. So, let $\gamma$ be a closed path in $\lambda^{-1}(\{1\})$ from $a$ to $a\left(a \in \mathbb{Z}^{n}\right)$ included in a unit square (if $n=2$ or $n=3$ and $\beta=26$ ), or in a unit cube. If the length of $\gamma$ is less than or equal to 2 then the property is obvious. Now, we assume that the length of $\gamma$ is greater than or equal to 3. We denote by $m$ the face in $\mathbb{F}^{n}$ laying at the centre of the unit square or cube $C$ including $\zeta(\gamma)$ and we observe that the $\operatorname{arc} \zeta(\gamma)$ is included in $m^{\uparrow}$. Since at least three facets of $C$ belongs to $\mu^{-1}(\{1\})$, we derive from Table 2 that, if $\alpha=3^{n}-1$ or ( $n=3$ and $\alpha=18)$, then $m \in \mu^{-1}(\{1\})$ and we can easily build ${ }^{10}$ a sequence of elementary stretchings from $(a)$ to $\zeta(\gamma)^{11}$. Hence, from now, we suppose that $\alpha=2 n$. To end the proof, we check all the loops $\gamma$ which can be drawn in a unit square or a unit cube in $\mathbb{Z}^{n}$ (up to rotations and symmetries). We can suppose without loss of generality that $\zeta(\gamma)$ is a simply closed arc in the sense that each face in $\zeta(\gamma)$ is passed through only once. The reader can check (in Figure 13) that in each case the $\operatorname{arc} \zeta(\gamma)$ can be deformed (in $\mu^{-1}(\{1\})$ ) in a constant arc by introducing $m$ in $\zeta(\gamma)$.

Lemma 4 Let $(\alpha, \beta)$ be a pair of adjacencies on $\mathbb{Z}^{n}$. Let $\varepsilon$ be the connectivity function associated to $(\alpha, \beta)$. Let $x \in\{0,1\}$. Let $\omega=\alpha$ if $x=1$ and $\omega=\beta$ if $x=0$. Let $\lambda: \mathbb{Z}^{n} \rightarrow\{0,1\}$ be an image in $\mathbb{Z}^{n}$ and $\mu=\zeta_{\varepsilon}(\lambda)$ be the corresponding image in $\mathbb{F}^{n}$. Let $a, b \in \mathbb{Z}^{n}$. Let $\chi, \chi^{\prime}$ be two arcs in $\mu^{-1}(\{x\})$ from $\dot{a}$ to $\dot{b}$ $\left(a, b \in \mathbb{Z}^{n}\right)$. If $\chi^{\prime}$ is an elementary stretching of $\chi$, then there exists in $\lambda^{-1}(\{x\})$ an $\omega$-path $\gamma$ from a to $b$ such that $\zeta(\gamma) \geqslant \chi$ and $\zeta(\gamma) \geqslant \chi^{\prime}$.

Proof There are two -very similar- cases in the definition of an elementary stretching, so we only provide the proof for one of them. Let $\chi=\left(f_{i}\right)_{i=0}^{r}(r \geq 1)$. Suppose that $\chi^{\prime}=$

\footnotetext{
10 We could also invoke the existence of a smallest element to derive the contractibility of $C \cap \lambda^{-1}(\{1\})$ (see, e.g., Corollary 8 in [37]) and thus its simple connectivity.

11 If $\zeta(\gamma)=\left(f_{i}\right)_{i=0}^{r}\left(r \geq 2\right.$ and $\left.f_{0}=f_{r}=a\right)$ we use the sequence of elementary stretchings $\left(f_{0}\right) \rightarrow\left(f_{0}, m, f_{r}\right) \rightarrow\left(f_{0}, m, f_{r-1}, f_{r}\right) \rightarrow \ldots \rightarrow$ $\left(f_{0}, m, f_{1}, \ldots, f_{r}\right) \rightarrow\left(f_{0}, f_{1}, \ldots, f_{r}\right)$.
} 


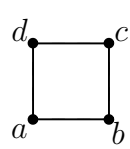

(a)

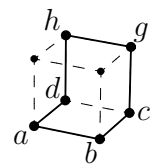

(b)

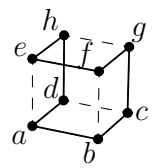

(c)

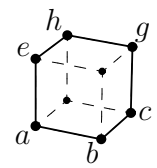

(d)
Fig. 13 We use the dual order in $\mathbb{F}^{n}$ to represent the unit square/cube: the facets are depicted by points, the $(n-1)$-faces by edges and so on (the face $m$ is then seen as the square $\{a, b, c, d\}$ or the cube $\{a, b, c, d, e, f, g, h\})$. The arc $\zeta(\gamma)$ is drawn like a solid black curve. Since $\zeta_{\varepsilon}(\lambda)$ is a regular image, whatever the connectivity function $\varepsilon$, when two $4 / 6$-adjacent vertices (which are actually facets) of the depicted polyhedron are on $\zeta(\gamma)$, the edge between them belongs to $\lambda^{-1}(\{1\})$ and when the four edges of a square are on $\zeta(\gamma)$ then the square between them belongs to $\lambda^{-1}(\{1\})$. (a) All the vertices, and thus $m$, are in $\lambda^{-1}(\{1\})$. (b, c, d) These three figures are only concerned by $(\alpha, \beta)=(6,18)$, i.e., $\varepsilon=(-1,1,-1)$. In each figure, the length of $\gamma$ is greater than or equal to 6 , so, from Table $2 m \in \lambda^{-1}(\{1\})$.

$\left(f_{i}\right)_{i=0}^{i_{0}} \cdot\left(f_{i_{0}}, f, f_{i_{0}+1}\right) \cdot\left(f_{i}\right)_{i=i_{0}+1}^{r}$ with $i_{0} \in \llbracket 0, r-1 \rrbracket$. We can assume without loss of generality that $f_{i_{0}} \subset f_{i_{0}+1}$. If $f \subset f_{i_{0}+1}$, from Proposition 16, we can build an $\omega$-path $\gamma$ in $\lambda^{-1}(\{x\})$ from $a$ to $b$ and two paths $p=\sum_{i=0}^{r} f_{i} \mathbf{1}_{I_{i}}$ and $q$ in $\mu^{-1}(\{x\})$ whose tracks are $\chi$ and $\zeta(\gamma)$ and such that $q \geqslant p$. We set $y=\max \left(I_{i_{0}}\right)=\inf \left(I_{i_{0}+1}\right)$ (since $f_{i_{0}} \subset f_{i_{0}+1}$, the interval $I_{i_{0}}$ is closed on the right (Proposition 13)). Let $\delta>0$ be a real such that $y-2 \delta \in I_{i_{0}}$ and $y+2 \delta \in I_{i_{0}+1}$ ( $\delta$ exists since $p$ is regular). We set $p^{\prime}=\sum_{i=0}^{i_{0}-1} f_{i} \mathbf{1}_{I_{i}}+f_{i_{0}} \mathbf{1}_{I}+f \mathbf{1}_{I^{\prime}}+f_{i_{0}+1} \mathbf{1}_{I^{\prime \prime}}+\sum_{i=i_{0}+2}^{r} f_{i} \mathbf{1}_{I_{i}}$ where $I^{\prime}=[y-\delta, y+\delta]$, if $f \subset f_{i_{0}}$ and $\left.\left.I^{\prime}=\right] y, y+\delta\right]$ if $f_{i_{0}} \subset f \subset f_{i_{0}+1}, I=I_{i_{0}} \backslash I^{\prime}$ and $I^{\prime \prime}=I_{i_{0}+1} \backslash I^{\prime}$. Proposition 13 ensures that $p^{\prime}$ is actually a path and it is plain that the track of $p^{\prime}$ is $\chi^{\prime}$ and $p^{\prime} \leqslant p$. We have thereby $p^{\prime} \leqslant p \leqslant q$. Hence, $\zeta(\gamma) \geqslant \chi$ and $\zeta(\gamma) \geqslant \chi^{\prime}$. If $f_{i_{0}+1} \subset f$, still using Proposition 16, we start to build an $\omega$-path $\gamma^{\prime}$ in $\lambda^{-1}(\{x\})$ from $a$ to $b$ and two paths $p^{\prime}=\sum_{i=0}^{i_{0}} f_{i} \mathbf{1}_{I_{i}}+f \mathbf{1}_{I}+\sum_{i=i_{0}+1}^{r} f_{i} \mathbf{1}_{I_{i}}, q^{\prime}$ in $\mu^{-1}(\{x\})$ whose tracks are $\chi^{\prime}$ and $\zeta\left(\gamma^{\prime}\right)$ and such that $q^{\prime} \geqslant p^{\prime}$. We set $p=\sum_{i=0}^{i_{0}} f_{i} \mathbf{1}_{I_{i}}+f_{i_{0}+1} \mathbf{1}_{I^{\prime}}+\sum_{i=i_{0}+2}^{r} f_{i} \mathbf{1}_{I_{i}}$ where $I^{\prime}=$ $I \cup I_{i_{0}+1}$. Then $p$ is a path whose track is $\chi$ and $p \leqslant p^{\prime} \leqslant q^{\prime}$. So, $\zeta\left(\gamma^{\prime}\right) \geqslant \chi$ and $\zeta\left(\gamma^{\prime}\right) \geqslant \chi^{\prime}$.

Lemma 5 Let $(\alpha, \beta)$ be a pair of adjacencies on $\mathbb{Z}^{n}$. Let $\varepsilon$ be the connectivity function associated to $(\alpha, \beta)$. Let $x \in\{0,1\}$. Let $\omega=\alpha$ if $x=1$ and $\omega=\beta$ if $x=0$. Let $\lambda: \mathbb{Z}^{n} \rightarrow\{0,1\}$ be an image in $\mathbb{Z}^{n}$ and $\mu=\zeta_{\varepsilon}(\lambda)$ be the corresponding image in $\mathbb{F}^{n}$. Let $a, b \in \mathbb{Z}^{n}$. Let $\chi$ be an arc from the facet $\dot{a}$ to the facet $\dot{b}$ in $\mu^{-1}(\{x\})$ and $\gamma, \gamma^{\prime}$ be two $\omega$-paths from a to $b$ such that $\zeta(\gamma) \geqslant \chi$ and $\zeta\left(\gamma^{\prime}\right) \geqslant \chi$. Then $\gamma$ and $\gamma^{\prime}$ are equivalent in $\lambda^{-1}(\{x\})$.

Proof Let $p, q, p^{\prime}, q^{\prime}$ be four paths in $\mu^{-1}(\{x\})$ whose tracks are respectively $\chi, \chi, \zeta(\gamma), \zeta\left(\gamma^{\prime}\right)$ and such that $p \leqslant p^{\prime}$ and $q \leqslant q^{\prime}$. If $p \neq q$, since these two regular finite paths have the same tracks, the intervals of their intervals sequences are homeomorphic (Proposition 13). Hence, there exists a homeomorphism $\varphi$, piecewise linear, such that $q=p \circ \varphi$. We set $p^{\prime \prime}=q^{\prime} \circ \varphi^{-1}$. As $q^{\prime} \geqslant q$, we have $p^{\prime \prime} \geqslant p$. Obviously, the track of $p^{\prime \prime}$ is $\zeta\left(\gamma^{\prime}\right)$. We set $p=\sum_{i=0}^{r} f_{i} \mathbf{1}_{I_{i}}, p^{\prime}=\sum_{i=0}^{s} g_{i} \mathbf{1}_{J_{i}}$ and $p^{\prime \prime}=\sum_{i=0}^{t} h_{i} \mathbf{1}_{K_{i}}(r, s, t \geq 0)$. We prove the lemma by induction on $r$, the size of $\chi$. For $r=0$, the result is obvious. Let us now suppose that the property is true whenever the size of $\chi$ is strictly lower than $r$ ( $r \geq 2$ for $r$ cannot be 1). Let $j \in \llbracket 1, r \rrbracket$ be the lowest integer such that $f_{j}$ is a local minimum of $\chi$, i.e., such that $f_{j-1} \supset f_{j} \subset f_{j+1}$ (remember that $f_{0}=\dot{a}$ and $f_{r}$ are facets) and let $j^{\prime} \in \llbracket j+1, r \rrbracket$ be the lowest integer such that $f_{j^{\prime}}$ is a local maximum of $\chi$. As $f_{j^{\prime}}$ is a local maximum, $I_{j^{\prime}}$ is an open interval of $[0,1]$ (Proposition 13). If $j^{\prime}=r$, that is $f_{j^{\prime}}=\dot{b}$, then $\zeta(\gamma)$ and $\zeta\left(\gamma^{\prime}\right)$ are included in $f_{j}^{\uparrow}$ so $\gamma$ and $\gamma^{\prime}$ are included in a same $2 \times 2$ square of $\mathbb{Z}^{n}$ (if $\operatorname{dim}\left(f_{j}\right) \geq n-2$ ) or in the same $2 \times 2 \times 2$ cube of $\mathbb{Z}^{n}$ (if $\left.\operatorname{dim}\left(f_{j}\right)=n-3\right)$. Moreover, if $n=3,(\alpha, \beta)=(6,26)$ (hence, $\varepsilon=-1$ ) and $\operatorname{dim}\left(f_{j}\right)=0$, from Proposition 1 , we derive that there is no facet with value $1-x$ in $f_{j}^{\uparrow}$. So, using one or the other version of the definition of digital equivalence, we can conclude that the arcs $\gamma$ and $\gamma^{\prime}$ are equivalent. Otherwise $\left(j^{\prime}<r\right)$, we set $\left.I_{j^{\prime}}=\right] y, z[$ with $0<y<z<1$. Let $k \in \llbracket 0, s \rrbracket$ and $k^{\prime} \in \llbracket 0, t \rrbracket$ be the even integers defined by $z \in J_{k} \cup J_{k+1}$ and $z \in K_{k^{\prime}} \cup K_{k^{\prime}+1}$. Since $p \leqslant p^{\prime}, p \leqslant p^{\prime \prime}$ and $g_{k}, h_{k^{\prime}} \in \mathbb{F}_{n}^{n}$ (in particular $J_{k}$ and $K_{k^{\prime}}$ are open on the left), this definition of $k$ and $k^{\prime}$ ensures that $g_{k} \supseteq f_{j^{\prime}} \subseteq h_{k^{\prime}}$ and thereby that $g_{i} \in f_{j}^{\uparrow}$ for all $i \leq k$ and $h_{i} \in f_{j}^{\uparrow}$ for all $i \leq k^{\prime}$. If $f_{j^{\prime}} \in \mathbb{F}_{n}^{n}$, then necessarily $q_{k}=h_{k^{\prime}}=f_{j^{\prime}}$ and we set $\chi_{0}^{\prime}=\left(f_{j^{\prime}}\right)$. Otherwise $\left(\operatorname{dim}\left(f_{j^{\prime}}\right)<n\right)$, we set $\chi_{0}^{\prime}=\left(g_{k}, f_{j^{\prime}}, h_{k^{\prime}}\right)$. In both cases, $\chi_{0}^{\prime}$ is an arc in $\mu^{-1}(\{x\})$ thus, from Proposition 16, we derive that there exists an $\omega$-path $\gamma_{0}^{\prime}$ in $\lambda^{-1}(\{x\})$ such that $\zeta\left(\gamma_{0}^{\prime}\right) \geqslant \chi_{0}^{\prime}$. We set $\zeta\left(\gamma_{0}^{\prime}\right)=\left(e_{i}\right)_{i=0}^{u}$ $(u \geq 0)$ and we have $e_{0}=g_{k}$ and $e_{u}=h_{k^{\prime}}$. Moreover $\chi_{0}^{\prime}$ is an arc in $f_{j^{\prime}}^{\uparrow}$ which is included in $f_{j}^{\uparrow}$ so $\zeta\left(\gamma_{0}^{\prime}\right)$ is also an arc in $f_{j}^{\uparrow}$. Let $\gamma_{0}, \gamma_{1}$ and $\gamma_{1}^{\prime}$ be the $\omega$-paths in $\lambda^{-1}(\{x\})$ such that $\zeta\left(\gamma_{0}\right)=\left(g_{i}\right)_{i=0}^{k}, \zeta\left(\gamma_{1}\right)=\left(g_{i}\right)_{i=k}^{s}$ and $\zeta\left(\gamma_{1}^{\prime}\right)=\left(h_{i}\right)_{i=k^{\prime}}^{t}$. We set $\gamma^{\prime \prime}=\gamma_{0} \cdot \gamma_{0}^{\prime} \cdot \gamma_{1}^{\prime}$. Since $\zeta\left(\gamma^{\prime \prime}\right)=\zeta\left(\gamma_{0}\right) \cdot \zeta\left(\gamma_{0}^{\prime}\right) \cdot\left(h_{k^{\prime}}, \ldots, h_{t}\right)$, it differs from $\zeta\left(\gamma^{\prime}\right)=\left(h_{0}, \ldots, h_{k^{\prime}}\right) .\left(h_{k^{\prime}}, \ldots, h_{t}\right)$ only in $f_{j}^{\uparrow}$. So, whatever is the adjacency relation, we can conclude as above that the arcs $\gamma^{\prime \prime}$ and $\gamma^{\prime}$ are equivalent.

In order to use the induction hypothesis, let us now define three new paths. (Of course, we still use the rule defined by Proposition 13 in order to get paths, this will not be indicated anymore.) Previously to the definition of the three paths, we need to choose a real $m \in] y, z\left[\cap\left(J_{k} \cup J_{k+1}\right) \cap\left(K_{k^{\prime}} \cup K_{k^{\prime}+1}\right)\right.$. Such a real exists since $J_{k} \cup J_{k+1}$ and $K_{k^{\prime}} \cup K_{k^{\prime}+1}$ are open on the left and contain $z$. The first path is obtained from $p$ by removing all faces before $f_{j^{\prime}}$ and replacing them by the facet $g_{k}: p_{1}=g_{k} \mathbf{1}_{[0, y[}+f_{j}^{\prime} \mathbf{1}_{[y, z[}+p_{\mid[z, 1]}$ where $p_{\mid I}$ means the restriction of the function $p$ to the interval $I$. Since we remove at least two faces $\left(f_{0}\right.$ and $\left.f_{j}\right)$ and add just one, the length of the track of $p_{1}$ is strictly lower than $r$. We must beware that all new paths are regular. This implies to check the first interval in $p_{[y, 1]}$. This interval is $[y, z[$ (remember that $] y, z\left[=I_{j^{\prime}}\right.$ ) and is not a singleton. The second path is 
obtained in a similar way from $p^{\prime}$ by removing all faces before $g_{k}$, so its track is $\zeta\left(\gamma_{1}\right): p_{1}^{\prime}=g_{k} \mathbf{1}_{[0, m[}+p_{[m, 1]}^{\prime}$. The first interval in $p_{[m, 1]}^{\prime}$ is either associated to $g_{k}$ (and then has to be merged with [0, $m\left[\right.$ ), or is associated to $g_{k+1}$ but in this case, by the definition of $k, z$ is also in this interval so the first interval can never be a singleton. The third path $p_{1}^{\prime \prime}$ is obtained from $p^{\prime \prime}$ by removing all faces before $h_{k^{\prime}}$ and putting in front of $h_{k^{\prime}}$ the faces of the arc $\chi_{0}^{\prime}$ with, for the choice of the intervals, the constraint that $p_{1}^{\prime \prime}$ must be equal to $p_{1}^{\prime}$ and $p_{1}$ on $\left[0, y\left[: p_{1}^{\prime \prime}=g_{k} \mathbf{1}_{[0, y[}+\sum_{i=1}^{u} e_{i} \mathbf{1}_{K_{i}^{\prime}}+p_{[m, 1]}^{\prime \prime}\right.\right.$ where the intervals $K_{i}^{\prime}$ are supposed to be well-defined. The track of $p_{1}^{\prime \prime}$ is $\zeta\left(\gamma_{0}^{\prime}\right) \cdot \zeta\left(\gamma_{1}^{\prime}\right)=\zeta\left(\gamma_{0}^{\prime} \cdot \gamma_{1}^{\prime}\right)$. The first interval in $p_{[m, 1]}^{\prime \prime}$ is either associated to $h_{k^{\prime}}=e_{u}$ (and then is to be merged with $K_{u}^{\prime}$ ), or is associated to $h_{k^{\prime}+1}$ but in this case, as above, $z$ is also in this interval so the first interval can never be a singleton. Hence, we have three regular paths $p_{1}, p_{1}^{\prime}, p_{1}^{\prime \prime}$ equal to $p, p^{\prime}, p^{\prime \prime}$ on $[m, 1]$ so we have $p_{1}^{\prime} \geqslant p_{1}$ and $p_{1}^{\prime \prime} \geqslant p_{1}$ on $[m, 1]$. The three paths are equal (to $g_{k}$ ) on $[0, y[$ and, on $\left[y, m\left[, p_{1}=f_{j^{\prime}}\right.\right.$ which is a face included in $p_{1}^{\prime}=g_{k}$ and in all the faces of $\chi_{0}^{\prime}$, the track of $p_{1}^{\prime \prime}$. Thus, $p_{1}^{\prime} \geqslant p_{1}$ and $p_{1}^{\prime \prime} \geqslant p_{1}$ on $[0,1]$. We can now apply the induction hypothesis: the $\omega$ paths $\gamma_{1}$ and $\gamma_{0}^{\prime} . \gamma_{1}^{\prime}$ are equivalent. Furthermore, the $\omega$-paths $\gamma=\gamma_{0} \cdot \gamma_{1}$ and $\gamma^{\prime \prime}=\gamma_{0} \cdot \gamma_{0}^{\prime} \cdot \gamma_{1}^{\prime}$ are equivalent. Since, we have proved before that $\gamma^{\prime \prime}$ and $\gamma^{\prime}$ are equivalent, we can eventually conclude that $\gamma$ and $\gamma^{\prime}$ are equivalent.

Proposition 18 Let $(\alpha, \beta)$ be a pair of adjacencies on $\mathbb{Z}^{n}$. Let $x \in\{0,1\}$. Let $\omega=\alpha$ if $x=1$ and $\omega=\beta$ if $x=0$. Let $\lambda: \mathbb{Z}^{n} \rightarrow\{0,1\}$ be an image in $\mathbb{Z}^{n}$. Let $a, b \in \mathbb{Z}^{n}$. Let $\gamma, \gamma^{\prime}$ be two $\omega$-paths from a to $b$ in $\lambda^{-1}(\{x\})$. If the arc $\zeta\left(\gamma^{\prime}\right)$ is a deformation in $\mu^{-1}(\{x\})$ of the arc $\zeta(\gamma)$, then $\gamma$ and $\gamma^{\prime}$ are equivalent in $\lambda^{-1}(\{x\})$.

Proof Let $\gamma, \gamma^{\prime}$ be two $\omega$-paths from $a$ to $b$ in $\lambda^{-1}(\{x\})$ such that $\zeta\left(\gamma^{\prime}\right)$ is a deformation of $\zeta(\gamma)$. By definition, there exist an integer $r \geq 0$ and a sequence $S$ of $\operatorname{arcs}$ in $\mu^{-1}(\{x\})$ (with $\mu=\zeta_{\varepsilon}(\lambda)$ where $\varepsilon$ is the connectivity function associated to $(\alpha, \beta)), S=\left(\chi_{0}=\zeta(\gamma), \ldots, \chi_{i}, \ldots, \chi_{r}=\zeta\left(\gamma^{\prime}\right)\right)$, such that, for each $i \in \llbracket 1, r \rrbracket$, either $\chi_{i}$ is an elementary stretching of $\chi_{i-1}$ or $\chi_{i-1}$ is an elementary stretching of $\chi_{i}$. From Lemma 4, we derive that there exists a sequence $S_{p}$ of $\omega$-paths in $\lambda^{-1}(\{x\})$, $S_{p}=\left(\gamma_{1}, \ldots, \gamma_{i}, \ldots, \gamma_{r}\right)$ such that for all $i \in \llbracket 1, r \rrbracket, \zeta\left(\gamma_{i}\right) \geqslant$ $\chi_{i-1}$ and $\zeta\left(\gamma_{i}\right) \geqslant \chi_{i}$. Then, from Lemma 5, we deduce the sequence of equivalence $\gamma \sim \gamma_{1} \sim \ldots \sim \gamma_{r} \sim \gamma^{\prime}$. So he have $\gamma \sim \gamma^{\prime}$.

Let $a \in \lambda^{-1}(\{x\})$. Let $\pi_{D}\left(\lambda^{-1}(\{x\}), a\right)$ be the digital fundamental group of $\lambda^{-1}(\{x\})$ with basepoint $a$ and $\rho\left(\mu^{-1}(\{x\}), \dot{a}\right)$ be the group of arcs in $\mu^{-1}(x)$ from $\dot{a}$ to $\dot{a}$, up to deformations.

From Propositions 14 and 17, we know that the function $\dot{\zeta}$ defined by

$$
\begin{aligned}
\dot{\zeta}: \quad \pi_{D}\left(\lambda^{-1}(x), a\right) & \rightarrow \rho\left(\mu^{-1}(x), \dot{a}\right) \\
{[\gamma] } & \mapsto \quad[\zeta(\gamma)]
\end{aligned}
$$

where $[y]$ denotes the equivalence class of $y$ (for the equivalence relation on digital paths of $\mathbb{Z}^{n}$ on the left side and for the deformation on arcs of $\mathbb{F}^{n}$ on the right side), is welldefined. Proposition 15 then states that $\dot{\zeta}$ is a morphism. Propositions 16 and 18 give the surjectivity and the injectivity of $\dot{\zeta}$, respectively. We conclude that the two groups $\pi_{D}\left(\lambda^{-1}(\{x\}), a\right)$ and $\rho\left(\mu^{-1}(\{x\}), \dot{a}\right)$ are isomorphic and, since $\rho\left(\mu^{-1}(\{x\}), \dot{a}\right)$ and $\pi\left(\mu^{-1}(\{x\}), \dot{a}\right)$ are isomorphic (Theorem 2), the following theorem holds.

Theorem 4 Let $(\alpha, \beta)$ be a pair of adjacencies on $\mathbb{Z}^{n}$. Let $\varepsilon$ be the connectivity function associated to $(\alpha, \beta)$. Let $\lambda$ : $\mathbb{Z}^{n} \rightarrow\{0,1\}$ be an image in $\mathbb{Z}^{n}$ and $\mu=\zeta_{\varepsilon}(\lambda)$ be the corresponding image in $\mathbb{F}^{n}$. For any $a \in \lambda^{-1}(\{x\})$, the digital fundamental group of $\lambda^{-1}(\{x\})$ with basepoint a is isomorphic to the fundamental group of the poset $\left(\mu^{-1}(\{x\}), \subseteq\right)$ with basepoint $\dot{a}$.

\section{Conclusion}

In this article, a modus operandi has been proposed to embed digital binary images, equipped with a pair of standard adjacencies, in the space of cubical complexes. In particular, it has been proved that it preserves the connected components of both object and background and preserves also the (digital) fundamental groups.

These results, associated to those proposed in [37], justify the soundness of all contributions previously devoted to design homotopy type-preserving binary image processing methods, especially concerning the correctness of their behaviour with respect to the "continuous" topology of the handled digital objects. They also permit to establish links between image processing/analysis methods developed either in classical digital spaces $\left(\mathbb{Z}^{n}\right)$ or cubical complexes $\left(\mathbb{F}^{n}\right)$, and to potentially unify some of them.

Processing binary digital images without topology alteration has been an active research field for several years. Nowadays, an increasing interest for the extension of this field to the case of colour/label images (i.e., not only binary images, but more generally $p$-ary ones) can be observed. Some applicative methods devoted to $p$-ary images have already been proposed [38-40]. However, the putative preservation of topological properties by such methods generally relies on ad hoc hypotheses or incompletely proved theoretical bases.

From a theoretical point of view, the issue of topology and topology preservation in digital $p$-ary images has not been intensively considered yet [41-46], and the proposed contributions still do not provide solutions in the most general cases in $\mathbb{Z}^{n}$ and $\mathbb{F}^{n}$. In this context, further works will now consist in extending the approach proposed in this article by defining a mapping enabling to embed $p$-ary images 


\section{A Auxiliary properties on complexes and posets}

Lemma 6 Let $f$, $g$ be two facets of $\mathbb{F}^{n}$. If $f \cap g \neq \emptyset$ then, for all $h \in \mathbb{F}^{n}$, we have $f \cap g<h \Rightarrow(h \subseteq f$ or $h \subseteq g)$.

Proof Set $f=\Pi_{i=1}^{n} f_{i}, g=\Pi_{i=1}^{n} g_{i}$ and $f \cap g=\Pi_{i=1}^{n} k_{i}$ with $f_{i}, g_{i} \in \mathbb{F}_{1}^{1}$ and $k_{i} \in \mathbb{F}_{1}^{1} \cup \mathbb{F}_{0}^{1}$ for all $i \in \llbracket 1, n \rrbracket$. Let $h=\Pi_{i=1}^{n} h_{i}$, with $h_{i} \in \mathbb{F}_{1}^{1} \cup \mathbb{F}_{0}^{1}$, be a face which covers $f \cap g$. Then, there exists $i_{0} \in \llbracket 1, n \rrbracket$ such that $h_{i_{0}} \in \mathbb{F}_{1}^{1}, k_{i_{0}} \in \mathbb{F}_{0}^{1}, f_{i_{0}} \cap g_{i_{0}}=k_{i_{0}} \subset h_{i_{0}}$ and, for all $i \neq i_{0}, k_{i}=h_{i}=f_{i} \cap g_{i}$. But, readily, there exist only two faces in $\mathbb{F}_{1}^{1}$ which include a given face of $\mathbb{F}_{0}^{1}$. Thus, $h_{i_{0}}=f_{i_{0}}$ or $h_{i_{0}}=g_{i_{0}}$ and, straightforwardly, $h \subseteq f$ or $h \subseteq g$.

Lemma 7 Let $0 \leq m<n$. Let $f \in \mathbb{F}_{m}^{n}$. Then:

(i) $\operatorname{Card}\left(f^{\uparrow+}\right)=2^{n-m}$.

(ii) For $\left\{g_{1}, g_{2}\right\} \in \operatorname{opp}(f) . f^{\uparrow+}=g_{1}^{\uparrow+} \sqcup g_{2}^{\uparrow+}$ (where $\sqcup$ denotes the disjoint union).

Proof (i) We can assume, without loss of generality, that $f=\prod_{i=1}^{n} I_{i}$ where $I_{i} \in \mathbb{F}_{1}^{1}$ if $i \leq m$ and $I_{i} \in \mathbb{F}_{0}^{1}$ otherwise. Obviously, for each $I_{i}$ $(m+1 \leq i \leq n)$ there are two sets in $\mathbb{F}_{1}^{1}$ including $I_{i}$ and therefore, there are $2^{n-m}$ products $\prod_{i=1}^{m} I_{i} \times \prod_{i=m+1}^{n} J_{i}$ where $I_{i} \subset J_{i} \in \mathbb{F}_{1}^{1}$ for each $i \in \llbracket m+1, n \rrbracket$.

(ii) We apply the first part of the lemma to the three faces $f, g_{1}, g_{2}$ : there are $2^{n-m}$ facets in $f^{\uparrow}$ and $2^{n-m-1}$ facets in $g_{1}^{\uparrow}$ as in $g_{2}^{\uparrow}$. Since, by definition of opp, $g_{1}^{\uparrow} \cap g_{2}^{\uparrow}=\emptyset$, we conclude that $f^{\uparrow+}=g_{1}^{\uparrow+} \sqcup g_{2}^{\uparrow+}$.

The following two lemmas use the definitions of $\mathrm{Card}^{-}$and $\mathrm{Card}^{+}$ given in Section 3.4.

Lemma 8 Let $f$ be a $k$-face in $\mathbb{F}^{n}$ (with $n-3 \leq k \leq n-1$ ). Let $r \in \llbracket 1,2^{n-k-1} \rrbracket$. Let $E$ be a set of facets in $f^{\uparrow}$ such that $\operatorname{Card}^{-}(E) \geq 2 r$. Then, there exist two faces $g, h \in \mathbb{F}^{n}$, opposite with respect to $f$ such that $\operatorname{Card}\left(E \cap g^{\uparrow}\right) \geq r$ and $\operatorname{Card}\left(E \cap h^{\uparrow}\right) \geq r$.

Proof We set $m=n-\operatorname{dim}(f)=n-k$. Thanks to the duality on the ordering in $\mathbb{F}^{n}$, the statement of this lemma is a consequence of the following one that we will prove: "if $f$ is a facet of $\mathbb{F}^{m}$ and $E$ is a set of $2 r 0$-faces in $f^{\downarrow}$, not in the configuration depicted in Figure 14(k), then there exists a facet $g$ of $f^{\downarrow \star}$ which contains exactly $r$ elements of $E$ ". Since the result is obvious if $m=1$ and we have restricted our lemma to $m \leq 3$, we can check all configurations, up to rotations, symmetries and duality (e.g., choosing six points among eight amounts to choose two of them). These configurations, in 2-D and 3-D, are depicted in Figure 14

and the only one, numbered (k), in which no facet of the boundary of $f^{\downarrow}$ contains half of the points corresponds to the configuration of a trihedron.

Lemma 9 Let $f$ be a $k$-face in $\mathbb{F}^{n}$ (with $n-3 \leq k \leq n-1$ ). Let $r \in \llbracket 1,2^{n-k-1} \rrbracket$. Let $E$ be a set of facets in $f^{\uparrow}$. If there exist $n-k$ faces which cover $f$ and whose stars contain at least $r$ facets of $E$, then $\operatorname{Card}^{+}(E) \geq 2 r-1$.

Proof As in the proof of Lemma 8, thanks to duality, we can replace the statement of this lemma by the following one that we will prove: "if $c$ is a facet of in $\mathbb{F}^{n}(1 \leq n \leq 3), E$ a set of 0 -faces in $c^{\downarrow}$ such that there exist $n$ facets of $c^{\downarrow \star}$ which contain, each, at least $r$ faces of $E(1 \leq$ $r \leq 2^{n-1}$ ), then $\operatorname{Card}(E) \geq 2 r-1$ or $r=3$ and $E$ is in the configuration depicted in Figure 15(b) (in which case, $\operatorname{Card}(E) \geq 2 r-2$ )". If $r=1$, the property is obvious, as is the case where two of the $n$ facets of $c^{\downarrow \star}$ which contain $r$ faces of $E$ are parallel (since two parallel faces do not share 0 -faces, in this case $\operatorname{Card}(E) \geq 2 r$ ). Hence, in the sequel we assume that all these $n$ facets share a same 0 -face. Moreover, it suffices to study minimal configurations, that is, sets $E$ which satisfy the hypothesis while none of their proper subsets does. If $n=2$ and $r=2$, there is only one such minimal configuration (up to rotations and symmetries), depicted in Figure 15(a). When $n=3$, let $b, f, l$ be the three facets of $c^{\downarrow \star}$ that contain $r$ faces of $E, t$ be the 0 -face contained in these three facets, and $x, y, z$ be the 0 -faces contained in exactly two of these three facets (in Figure 15(b-e), $b, f, l$ are located at the bottom, front and left positions, respectively). If $r=2$ the first two 0 -faces of $E$ contained in $b$ cannot be shared with $f$ and $l$ (but can be shared with either $f$ or $l$ ). Thus, there must exist at least a third face of $E$ in $f$ or $l$, implying that $\operatorname{Card}(E) \geq 3$. If $r=3$, the only minimal configuration with $\{t, x, y, z\} \subseteq E$ is depicted in Figure 15(b) and the only minimal configuration, up to rotations, with $t \in E$ and $\operatorname{Card}(\{x, y, z\} \cap E)=2$ is depicted on Figure 15(c). The case $\operatorname{Card}(\{x, y, z\} \cap E)=1$ is not possible. The only minimal configuration with $t \notin E$ is depicted on Figure 15(d). If $r=4$, the only minimal configuration is depicted on Figure 15(e). The reader can then easily check from Figure 15(b-e) that the statement of the lemma is true.

Lemma 10 Let $(T, \leqslant)$ be a poset. The path product is compatible with the order relation: for any $a, b, c \in T$ and any paths $p \leqslant p^{\prime}$ from $a$ to $b$ and $q \leqslant q^{\prime}$ from b to $c$, we have $p . q \leqslant p^{\prime} . q^{\prime}$.

Proof Let $t \in[0,1]$. If $t \leq \frac{1}{2}$, then, $p \cdot q(t)=p(2 t) \leqslant p^{\prime}(2 t)=p^{\prime} \cdot q^{\prime}(t)$. If $t \geq \frac{1}{2}$, then $p \cdot q(t)=q(2 t-1) \leqslant q^{\prime}(2 t-1)=p^{\prime} \cdot q^{\prime}(t)$. 


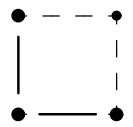

(a)

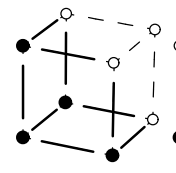

(b)

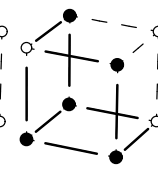

(c)

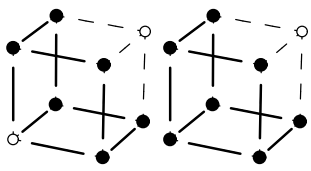

(d)

(e)
Fig. 15 Configurations used in the proof of Lemma 9 (see text). Black points: faces of $E$. Black edges: edges of three facets of the cube that contain at least $r$ faces of $E$. (a) $n=2$ and $r=2$. (b-d) $n=3$ and $r=3$. (e) $n=3$ and $r=4$.

\section{References}

1. A. Rosenfeld, J. L. Pfaltz, Sequential operations in digital picture processing, Journal of the Association for Computer Machinery 13 (4) (1966) 471-494.

2. O. Duda, P. E. Hart, J. H. Munson, Graphical data processing research study and experimental investigation, Tech. Rep. AD650926, Stanford Research Institute (1967).

3. A. Rosenfeld, Connectivity in digital pictures, Journal of the Association for Computer Machinery 17 (1) (1970) 146-160.

4. T. Y. Kong, A digital fundamental group, Computers and Graphics 13 (2) (1989) 159-166.

5. T. Y. Kong, A. Rosenfeld, Digital topology: introduction and survey, Computer Vision, Graphics and Image Processing 48 (3) (1989) 357-393.

6. E. Khalimsky, R. Kopperman, P. R. Meyer, Computer graphics and connected topologies on finite ordered sets, Topology and its Applications 36 (1) (1990) 1-17.

7. V. A. Kovalevsky, Finite topology as applied to image analysis, Computer Vision, Graphics, and Image Processing 46 (2) (1989) 141-161.

8. G. Bertrand, New notions for discrete topology, in: Discrete Geometry for Computer Imagery - DGCI 1999, Vol. 1568 of Lecture Notes in Computer Science, Springer, 1999, pp. 218-228.

9. G. Bertrand, G. Malandain, A new characterization of threedimensional simple points, Pattern Recognition Letters 15 (2) (1994) 169-175.

10. G. Bertrand, A Boolean characterization of three-dimensional simple points, Pattern Recognition Letters 17 (2) (1996) 115-124.

11. S. Fourey, R. Malgouyres, A concise characterization of 3D simple points, Discrete Applied Mathematics 125 (1) (2003) 59-80.

12. M. Couprie, G. Bertrand, New characterizations of simple points in 2D, 3D and 4D discrete spaces, IEEE Transactions on Pattern Analysis and Machine Intelligence 31 (4) (2009) 637-648.

13. Y. Cointepas, I. Bloch, L. Garnero, Cellular complexes: A tool for 3D homotopic segmentation in brain images, in: International Conference on Image Processing - ICIP 1998, Vol. 3, 1998, pp. 832-836.

14. X. Daragon, M. Couprie, Segmentation du néo-cortex cérébral depuis des données IRM dans le cadre de la topologie des ordres, in: Reconnaissance de Formes et Intelligence Artificielle - RFIA 2002, Vol. 3, 2002, pp. 809-818.

15. G. Bertrand, M. Couprie, Two-dimensional thinning algorithms based on critical kernels, Journal of Mathematical Imaging and Vision 31 (1) (2008) 35-56.

16. X. Han, C. Xu, J. L. Prince, A topology preserving level set method for geometric deformable models, IEEE Transactions on Pattern Analysis and Machine Intelligence 25 (6) (2003) 755-768.
17. Y. Bai, X. Han, J. L. Prince, Topology-preserving geometric deformable model on adaptive quadtree grid, in: Computer Vision and Pattern Recognition - CVPR 2007, 2007, pp. 1-8.

18. J.-F. Mangin, V. Frouin, I. Bloch, J. Régis, J. López-Krahe, From $3 \mathrm{D}$ magnetic resonance images to structural representations of the cortex topography using topology preserving deformations, Journal of Mathematical Imaging and Vision 5 (4) (1995) 297-318.

19. P. Dokládal, C. Lohou, L. Perroton, G. Bertrand, Liver blood vessels extraction by a 3-D topological approach, in: Medical Image Computing and Computer-Assisted Intervention - MICCAI 1999, Vol. 1679 of Lecture Notes in Computer Science, Springer, 1999, pp. $98-105$.

20. Z. Aktouf, G. Bertrand, L. Perroton, A three-dimensional holes closing algorithm, Pattern Recognition Letters 23 (5) (2002) 523531.

21. N. Passat, C. Ronse, J. Baruthio, J.-P. Armspach, M. Bosc, J. Foucher, Using multimodal MR data for segmentation and topology recovery of the cerebral superficial venous tree, in: International Symposium on Visual Computing - ISVC 2005, Vol. 3804 of Lecture Notes in Computer Science, Springer, 2005, pp. 60-67.

22. S. Faisan, N. Passat, V. Noblet, R. Chabrier, C. Meyer, Topologypreserving warping of binary images according to one-to-one mappings, IEEE Transactions on Image Processing To appear.

23. T. Y. Kong, A. W. Roscoe, A theory of binary digital images, Computer Vision, Graphics and Image Processing 32 (2) (1985) 221243.

24. R. Ayala, E. Domínguez, A. Francés, A. Quintero, Digital lighting functions, in: Discrete Geometry for Computer Imagery - DGCI 1997, Vol. 1347 of Lecture Notes in Computer Science, Springer, 1997, pp. 139-150.

25. T. Y. Kong, Topology-preserving deletion of 1's from 2-, 3- and 4dimensional binary images, in: Discrete Geometry for Computer Imagery - DGCI 1997, Vol. 1347 of Lecture Notes in Computer Science, Springer, 1997, pp. 3-18.

26. G. Bertrand, M. Couprie, A model for digital topology, in: Discrete Geometry for Computer Imagery - DGCI 1999, Vol. 1568 of Lecture Notes in Computer Science, Springer, 1999, pp. 229-241.

27. R. Ayala, E. Domínguez, A. R. Francés, A. Quintero, Homotopy in digital space, in: Discrete Geometry for Computer Imagery DGCI 2000, Vol. 1953 of Lecture Notes in Computer Science, Springer, 2000, pp. 3-14.

28. R. Ayala, E. Domínguez, A. R. Francés, A. Quintero, Digital homotopy with obstacles, Discrete Applied Mathematics 139 (1-3) (2004) 5-30.

29. G. Birkhoff, Lattice Theory, 8th Edition, Vol. 25 of American Mathematical Society Colloquium Publications, American Mathematical Society, Providence, RI, 1995.

30. T. S. Blyth, Lattices and Ordered Algebraic Structures, Springer, London, 2005.

31. J. Munkres, Topology, Prentice Hall, 1999.

32. O. Y. Viro, O. A. Ivanov, N. Y. Netsvetaev, V. M. Kharlamov, Elementary Topology: Problem Textbook, AMS, 2008.

33. C. R. F. Maunder, Algebraic Topology, Dover, 1996.

34. A. May, A Concise Course in Algebraic Topology, U. Chicago Press, 1999.

35. A. Hatcher, Algebraic Topology, Cambridge University Press, 2002.

36. N. Passat, L. Mazo, An introduction to simple sets, Pattern Recognition Letters 30 (15) (2009) 1366-1377.

37. L. Mazo, N. Passat, M. Couprie, C. Ronse, Paths, homotopy and reduction in digital images, Tech. Rep. hal-00512228, Université Paris-Est, (Submitted) (2010).

URL http://hal.archives-ouvertes.fr/hal-00512228/ $\mathrm{fr} /$ 
38. F. Poupon, J.-F. Mangin, D. Hasboun, C. Poupon, I. E. Magnin, V. Frouin, Multi-object deformable templates dedicated to the segmentation of brain deep structures, in: International Conference on Medical Image Computing and Computer Assisted Intervention MICCAI 1998, Vol. 1496 of Lecture Notes in Computer Science, Springer, 1998, pp. 1134-1143.

39. P.-L. Bazin, D. L. Pham, Topology-preserving tissue classification of magnetic resonance brain images, IEEE Transactions on Medical Imaging 26 (4) (2007) 487-496.

40. S. Miri, N. Passat, J.-P. Armspach, Topology-preserving discrete deformable model: Application to multi-segmentation of brain MRI, in: International Conference on Image and Signal Processing - ICISP 2008, Vol. 5099 of Lecture Notes in Computer Science, Springer, 2008, pp. 67-75.

41. L. J. Latecki, 3D well-composed pictures, Graphical Models and Image Processing 59 (3) (1997) 164-172.

42. F. Ségonne, J.-P. Pons, E. Grimson, B. Fischl, A novel level set framework for the segmentation of medical images under topology control, in: ICCV Workshop on Computer Vision for Biomedical Image Applications, 2005, pp. 135-145.

43. P.-L. Bazin, L. M. Ellingsen, D. L. Pham, Digital homeomorphisms in deformable registration, in: Information Processing in Medical Imaging - IPMI 2007, Vol. 4584 of Lecture Notes in Computer Science, Springer, 2007, pp. 211-222.

44. M. Siqueira, L. J. Latecki, N. Tustison, J. Gallier, J. Gee, Topological repairing of 3D digital images, Journal of Mathematical Imaging and Vision 30 (3) (2008) 249-274.

45. G. Damiand, Topological model for 3D image representation: Definition and incremental extraction algorithm, Computer Vision and Image Understanding 109 (3) (2008) 260-289.

46. A. Dupas, G. Damiand, J.-O. Lachaud, Multi-label simple points definition for 3D images digital deformable model, in: Discrete Geometry for Computer Imagery - DGCI 2009, Vol. 5810 of Lecture Notes in Computer Science, Springer, 2009, pp. 218-229. 